Article

\title{
Can Precision Agriculture Increase the Profitability and Sustainability of the Production of Potatoes and Olives?
}

\author{
Frits K. van Evert ${ }^{1, *}$ (D) , Daniel Gaitán-Cremaschi ${ }^{2}$, Spyros Fountas ${ }^{3}$ and Corné Kempenaar ${ }^{1,4}$ \\ 1 Agrosystems Research, Wageningen University \& Research, P.O. Box 16, 6700 AA Wageningen, \\ The Netherlands; corne.kempenaar@wur.nl \\ 2 Farming Systems Ecology, Wageningen University \& Research, P.O. Box 430, 6700 AK Wageningen, \\ The Netherlands; daniel.gaitancremaschi@wur.nl \\ 3 Natural Resources Management and Agricultural Engineering, Agricultural University of Athens, \\ 11855 Athens, Greece; spyros.fountas@aua.gr \\ 4 Team Applied Research, Aeres University of Applied Sciences, De Drieslag 4, 8251 JZ Dronten, \\ The Netherlands \\ * Correspondence: frits.vanevert@wur.nl; Tel.: +31-317-480573
}

Received: 24 September 2017; Accepted: 13 October 2017; Published: 17 October 2017

\begin{abstract}
For farmers, the application of Precision Agriculture (PA) technology is expected to lead to an increase in profitability. For society, PA is expected to lead to increased sustainability. The objective of this paper is to determine for a number of common PA practices how much they increase profitability and sustainability. For potato production in The Netherlands, we considered variable rate application (VRA) of soil herbicide, fungicide for late blight control, sidedress $\mathrm{N}$, and haulm killing herbicide. For olive production in Greece, we considered spatially variable application of $\mathrm{P}$ and $\mathrm{K}$ fertilizer and lime. For each of the above scenarios, we quantified the value of outputs, the cost of inputs, and the environmental costs. This allowed us to calculate profit as well as social profit, where the latter is defined as revenues minus conventional costs minus the external costs of production. Social profit can be considered an overall measure of sustainability. Our calculations show that PA in potatoes increases profit by $21 \%\left(420 € \mathrm{ha}^{-1}\right)$ and social profit by $26 \%$. In olives, VRA application of $\mathrm{P}, \mathrm{K}$, and lime leads to a strong reduction in nutrient use and although this leads to an increase in sustainability, it has only a small effect on profit and on social profit. In conclusion, PA increases sustainability in olives and both profitability and sustainability in potatoes.
\end{abstract}

Keywords: potato; olive; social profit; variable rate application

\section{Introduction}

Rising input costs and increasing societal demand for sustainability, call for significant improvements in resource use efficiency in agriculture. Fortunately, in many cases resources such as fertilizers, pesticides, irrigation water, land, and labor can be used with much greater efficiency than currently found by measuring and responding to spatial and temporal variability. Precision Agriculture (PA) is the scientific domain that deals with management of spatial and temporal variability to improve economic returns and reduce environmental impact. For farmers, PA is expected to lead to an increase in profitability; for society, PA is expected to lead to increased sustainability [1-3].

PA techniques can be classified as recording, reacting, or guidance techniques [4]. A recording technique measures the status of crop, soil, weeds, pests, or diseases, typically using sensors on mobile equipment. Examples are soil scans, canopy reflectance measurements, weed detection using cameras, yield monitors on grain combines or potato harvesters, and sensors mounted on satellites, airplanes and Unmanned Aerial Vehicles (UAVs) [5,6]. Typical examples of reacting techniques are variable rate 
application of fertilizers and pesticides, usually on the basis of prepared maps, but this can also be on-the-go in response to a sensor signal. The most ubiquitous guidance technique is Global Navigation Satellite System (GNSS) based auto-steering for tractors and other machinery which reduces overlap between tractor passes and reduces operator fatigue [7]. Guidance techniques are also the foundation of Controlled Traffic Farming (CTF).

Recording techniques and reacting techniques as described above must be linked by a data processing step in which an action is defined based on the new data, previously recorded data, data from other sources (weather), scientific knowledge (mechanistic models), practical knowledge (empirical models), and farmer preferences. Typically, a Farm Management Information System (FMIS) is used to handle the data [8]. The most advanced FMIS include algorithms to make decisions. For example, Akkerweb (www.akkerweb.nl), which evolved out of a decision support system for control of plant parasitic nematodes [9,10], has more than ten recommendation apps [11,12]. A recent review of data recording and processing for weed control and crop protection is available [13].

PA can be used to make actions spatially precise. Conventionally, fertilizer or pesticide is applied uniformly on the entire field. Against this baseline, PA can increase fertilizer efficiency by measuring crop nutrient status and adjusting the fertilizer rate accordingly. Also, PA can increase herbicide use efficiency by adjusting the herbicide application rate to the observed weed density. The scale at which measurements are made and at which actions are implemented is important [14]: in general, the reduction in fertilizer or pesticide use will be greater as the area for which decisions are made decreases. Thus, recording and reacting on $5 \times 5 \mathrm{~m}^{2}$ will often result in greater reductions than recording and reacting on $50 \times 50 \mathrm{~m}^{2}$ but working on a smaller scale will require a larger investment in machines [15].

PA can also be used to make actions temporally precise. In this mode, a decision is made when (if at all) to perform an operation, for example to control weeds [16,17], to control late blight [18], or to control plant parasitic nematodes [9].

PA is thought to benefit both the farmer and society. For farmers, PA is attractive primarily if it provides a positive return on investment. Switching from conventional agriculture to PA requires investments: in technology (sensors, software, applicators), in knowledge (to operate the new machinery), and time (to learn a different way of working, to build relationships with a new set of service providers). The return on investment may come from several directions. Typically, PA will result in a reduction in input use. For expensive inputs such as certain pesticides, the associated cost savings may provide a sufficient return on investment. This will not be the case for cheap inputs such as nitrogen $(\mathrm{N})$ fertilizer. Here, PA may be worthwhile for a farmer because it makes it easier to comply with laws that regulate fertilizer use. PA may lead to yield increase and thus higher revenue, for example because a reduced herbicide application causes less damage to the crop. PA may increase revenue through quality increase, for example when variable-rate planting or variable-rate application of $\mathrm{N}$ in potatoes leads to more uniform tuber size distribution or higher specific gravity of tubers.

For society, PA is attractive because it is assumed to increase the sustainability of farming. This is a reasonable assumption because in many cases a reduction in the use of inputs will lead to a reduction in environmental impact, but this is not necessarily the case. As an example, let us consider weed control. Weed control using an herbicide has a negative impact on the environment. This impact may be avoided by using close-to-crop mechanical weeding guided by computer vision. However, $\mathrm{CO}_{2}$ emission of such a system is higher because mechanical weeding requires more energy than is needed to produce and apply an herbicide, especially if two or more weeding passes are required. Thus the question arises whether sustainability is improved by accepting higher $\mathrm{CO}_{2}$ emissions in order to achieve a reduction in herbicide use.

The Brundtland Commission provided a widely accepted, qualitative definition of sustainability when it stated that "sustainable development is development that meets the needs of the present without compromising the ability of future generations to meet their own needs" [19]. Many other definitions can be found [20-24]. These definitions have as a common thread that three categories 
of aspects are considered, namely economics ("profit"), environment ("planet"), and well-being of humans ("people"), the so-called Triple Bottom Line [25,26]. Thus, sustainability measurement requires that trade-offs between categories are quantified, something that is not normally in the mind set of people [27].

Life Cycle Analysis (LCA) is one of the concrete methodologies that recognizes the Triple Bottom Line and it is widely used to assess sustainability in concrete cases [28-30]. LCA measures performance in impact categories such as global warming, acidification, eco-toxicity, but it stops short of integrating these separate measures into one composite indicator. Such an overall measure of sustainability is provided by indicator-based methods [31,32], e.g., Multi Criteria Analysis (MCA) [33], Total Factor Productivity (TFP) [34-37] and indexes of sustainability [38,39]. In this paper we focus on the adjusted (or social) profit method [40].

The objective of this paper is to examine several PA practices in potato and olive production in terms of how much they increase profitability and sustainability.

\section{Materials and Methods}

Below we describe social profit as a method to aggregate various aspects of sustainability into one number, with the aim of enabling comparisons between agricultural systems. We describe the concept of social profit and how it is calculated, and next describe which methods we used to calculate a price for externalities.

\subsection{Social Profit: Theoretical Framework}

Consider an agricultural production process where $M$ outputs are produced while using $N$ inputs. This process can be described by:

\begin{tabular}{cc}
\hline$M$ outputs & $y=\left(y_{1}, y_{2}, \ldots y_{M}\right) \in \Re_{+}^{M}$, \\
$N$ inputs & $x=\left(x_{1}, x_{2}, \ldots x_{N}\right) \in \Re_{+}^{N}$ \\
Output prices & $p=\left(p_{1}, p_{2}, \ldots p_{M}\right) \in \Re_{+}^{M}$ \\
Input prices & $w=\left(w_{1}, w_{2}, \ldots w_{N}\right) \in \Re_{+}^{N}$ \\
\hline
\end{tabular}

where $y$ is the vector of output quantities, $x$ is the vector of input quantities, $p$ is the vector of output prices, $w$ is the vector of input prices.

Then, profit $(P)$ is defined as the difference between revenues $\left(p^{\prime} y\right)$ (the value of outputs) and conventional costs $\left(w^{\prime} x\right)$ (the value of inputs):

$$
P=p^{\prime} y-w^{\prime} x
$$

As side effects of production, multiple environmental impacts (the so-called externalities) are produced. Examples include water and soil pollution, greenhouse gas emissions, and loss of biodiversity. We assume that externalities can be priced. This is described as follows:

\begin{tabular}{cc}
\hline$J$ negative externalities & $b=\left(b_{1}, b_{2}, \ldots b_{J}\right) \in \Re_{+\prime}^{J}$ \\
Externality prices & $r=\left(r w_{1}, r w_{2}, \ldots r_{N}\right) \in \Re_{+}^{J}$ \\
\hline
\end{tabular}

where $b$ is the vector of quantities of externalities and $r$ is the vector of prices of externalities.

Then, social profit $(S P)$ is defined as the net social value of the agricultural production process, that is the profits (defined in Equation (1)) minus the external costs of production $\left(r^{\prime} b\right)$ :

$$
S P=p^{\prime} y-w^{\prime} x-r^{\prime} b
$$




\subsection{Determining the Price of Externalities}

In order to calculate social profit, the price of negative externalities must be known or assumed. In this paper we concern ourselves with the following externalities: emission of greenhouse gases (GHG), expressed as $\mathrm{CO}_{2}$-equivalents; pesticide toxicity, expressed using the Environmental Impact Quotient (EIQ) model; loss of biodiversity resulting from the application of fertilizers; and eutrophication of surface waters resulting from leaching of nutrients, expressed as kilograms of leached nutrient $(\mathrm{N}$ and $\mathrm{P})$.

For $\mathrm{CO}_{2}$ emissions, there is a more-or-less functioning emissions trading scheme and this gives rise to a market price. We set the cost of emitting one ton of $\mathrm{CO}_{2}$ to 31 USD [41]. The other three externalities mentioned are not traded in well-defined markets and market price information does not exist. For such cases, a number of methods exist that aim to estimate the economic value of the externality and calculate a so-called shadow price. Shadow prices for the three externalities were transferred from existing actual cost based studies found in literature sources. Shadow prices were adjusted to the national context using the ratio of the Gross Domestic Product (GDP) per capita of The Netherlands (for potatoes) and Greece (for olives) to the average GDP per capita of the place where the estimation was made, expressed in purchasing power parities (PPP). In doing so, it is assumed that the Willingness to Pay (WTP) to avoid or to mitigate the damages is proportional to the per capita income of each country [42] and is locally determined.

The externality pesticide toxicity was estimated using the Environmental Impact Quotient (EIQ) model which was specifically developed to provide an assessment of the risks involved with biocide use [43]. As such, EIQ does not provide exact answers to what the impact of biocide application would be, but allows comparison of potential impacts from different farm management practices regarding the use of biocides. It does so by giving an EIQ score to each active ingredient (a.i.) in three components: (1) an EIQ score in the environmental component that is based on the impact of the active ingredient on aquatic organisms, bees, birds, and beneficial insects; (2) an EIQ score in the farm worker component that reflects the impact on applicators and pickers; and (3) an EIQ score in the consumer component based on consumer exposure and potential groundwater effects $[43,44]$. The total EIQ score is calculated as the average of the three components and reflects the overall toxicity of each pesticide active ingredient. To estimate the toxicity of the weed, pest and disease control strategy in conventional operations and when using precision agriculture practices, the total EIQ score of each of the pesticide active ingredients used at each of the production systems was multiplied by its application rate (kilograms of a.i.). The EIQ scores were summed over all used active ingredients at the conventional and alternative production systems, yielding the externality pesticide toxicity. The price of one unit of EIQ is elaborated by [37].

We followed earlier work to price eutrophication and loss of biodiversity [35], which itself is based on the estimated environmental cost of the use of pesticides and of nutrient leaching nationwide in the United Kingdom [45-48].

\section{Cases}

Below, we describe cases and quantify physical inputs, physical outputs, externalities, and calculate social profit for each case, both in conventional operation and when using precision agriculture.

\subsection{Potatoes in The Netherlands}

Potato (Solanum tuberosum L.) is the most important arable crop in The Netherlands. It is grown on ca. $20 \%$ of the arable land, with a total production of 7.5 million $\mathrm{Mg} \mathrm{y}^{-1}$ and a farm-level value of $€ 800$ million [49]. We focus on four precision agriculture operations in potatoes, namely (i) variable rate application of soil herbicides, (ii) fungicides to control late blight, (iii) sidedress $\mathrm{N}$, and (iv) potato haulm killing (PHK) herbicides. 


\subsubsection{Baseline}

We used standard tables on agricultural budgeting and practices ("KWIN") [50] to list quantities and values of inputs and outputs in potato production. We focused on ware potatoes grown on the fertile clay soil of Flevoland province. KWIN lists inputs and outputs needed for 1 ha of potatoes but it excludes the cost of the farmer's own labor, the cost of using the farm's own machines, and the cost of the farm's capital. The value of these items cannot be ascertained straightforwardly as it is highly dependent on the specific farm. We assumed that the value of the farmer's labor and the farm's machines is one-third of the value of the potatoes produced [51].

\subsubsection{Soil Herbicide}

A soil herbicide is applied to the soil and kills weeds when the herbicide is taken up through the roots. Soil herbicides often play an important role in weed control strategies in conventional arable farming and are typically applied uniformly on the field. The amount of soil herbicides can be reduced by adjusting the dosage to the local soil condition. In particular, soil herbicides are more effective in areas where soil organic matter content is low; thus, the application rate of soil herbicides can be lowered in those areas without affecting their efficacy. Trials with VRA soil herbicides are listed in Table 1 . Reductions in herbicide use between $20 \%$ and $40 \%$ were reported.

In this paper, we assumed that an average reduction of $27 \%$ of herbicide use can be achieved (Table 1 ). To be considered is the additional cost in making a map of soil organic matter content. We assume that this is done using a VERIS MSP3 soil scan (http:/ / veristech.com/the-sensors/msp3). This needs to be done once every three years at a cost of $150 € \mathrm{ha}^{-1}$ each time resulting in an annual cost of 50 euro ha $^{-1}$. A regular herbicide sprayer can be used so there is no additional cost for precision application equipment. The baseline cost of the herbicide is $76 € \mathrm{ha}^{-1}$ and the savings are $0.27 \times 76=20 € \mathrm{ha}^{-1}$. An additional benefit of a reduction in soil herbicide use is that crop damage is reduced, leading to crop yield increases between $0 \%$ and $5 \%$. Here we assume a yield increase of $3 \%$.

\subsubsection{Late Blight}

Late blight in potatoes is caused by the oomycete Phytophthora infestans. Potato crops are highly susceptible to infection by this pathogen. Infection is favored by wet environmental conditions, such as high relative humidity and rain. To prevent infection and damage, farmers apply fungicides circa every 5 to 10 days and 10 to 15 sprays per growth season. Farmers mainly apply fungicides with a preventive mode of action. The timing of fungicide sprays can be based on decision support systems that predict infection periods [52]. Fungicide dose of sprays can be based on the aboveground amount of leaves and stems, as measured with crop reflection sensors. The more biomass present on a part of the field, the higher the dose of the fungicide should be [11]. Trials with VRA fungicides in potatoes are listed in Table 2. Reductions in herbicide use between $20 \%$ and $30 \%$ were reported.

In this paper, we assumed that an average reduction of $22 \%$ of fungicide use can be achieved (Table 2). There is the additional cost of obtaining biomass maps and some extra decision support. We assume that this is done using a Yara N-Sensor or GreenSeeker sensors on SensiSpray $[11,53]$. We assume that a $\mathrm{N}$-Sensor costs 25,000 euro. We further assume that the sensor is fully depreciated in 5 years, that it is used on 100 ha of potatoes each year, and that half of its use is in other crops. Then the price per ha is $25,000 / 5 / 100 / 2=25 € \mathrm{ha}^{-1} \mathrm{y}^{-1}$.

A regular fungicide sprayer can be used so there is no additional cost for precision application equipment. Fungicide products applied were Shirlan, Revus, and Infinito. The baseline cost of the fungicides are $383 € \mathrm{ha}^{-1}$ and the savings are $0.22 \times 383=84 € \mathrm{ha}^{-1}$. We do not assume an effect on the yield of the crop. 


\subsubsection{Sidedress $N$}

In the temperate climate of the Netherlands, soil nitrogen supply varies widely from year to year and from field to field. Potato farmers can respond to the corresponding variability in $\mathrm{N}$ fertilizer requirement by measuring the time course of nitrogen uptake and applying an appropriate amount of sidedress $\mathrm{N}$ if and when needed. Sidedress recommendations based on physical measurements of crop and/or soil are commonly used [54], but the cost of sampling and the time required for analysis of samples are considered limitations of these methods. Also, at most, a few samples are taken per field, so the spatial resolution of this method is limited. In contrast, optical measurement of crop N status is quick, cheap, and can be done on every square meter of a field thus enabling variable rate application (VRA) of $\mathrm{N}$ [55]. In the Netherlands, development of a crop reflectance-based N sidedress system for potatoes started nearly 20 years ago [56] and was fully documented recently [57]. Trials are listed in Table 3. With reflectance-based $\mathrm{N}$ sidedress, $\mathrm{N}$ use can be reduced $15 \%$ on average without affecting yield or quality.

We made the following assumptions. The Yara $\mathrm{N}$-Sensor has been demonstrated to perform well. We assumed that a $\mathrm{N}$-Sensor costs 25,000 euro. We further assumed that the sensor is fully depreciated in 5 years, that it is used on 100 ha of potatoes each year, and that half of its use is in other crops. Then the price per ha is $25,000 / 5 / 100 / 2=25 € \mathrm{ha}^{-1} \mathrm{y}^{-1}$.

Standard $N$ application rate $=250 \mathrm{~kg} \mathrm{ha}^{-1}$, so saving can be $37.5 \mathrm{~kg} \mathrm{~N} \mathrm{ha}^{-1}$ which equals $38 € \mathrm{ha}^{-1}$.

We assumed that every $\mathrm{kg} \mathrm{N}$ that is applied, but not used by the crop, leaches to the groundwater. Thus the reduction in leaching is also $37.5 \mathrm{~kg} \mathrm{~N} \mathrm{ha}^{-1}$.

\subsubsection{Potato Haulm Killing (PHK)}

In order to harvest potato crops mechanically, the aboveground canopy has to be killed circa 3 weeks before the harvest date. Otherwise, the skin of the tubers is not yet hardened and damage during harvest will occur. The most common method to kill the potato canopy before harvest is use of defoliant herbicides [58]. Herbicide dose can be based on the aboveground amount and activity of the biomass, as measured with crop reflectance sensors. The more biomass present on a part of the field, the higher the dose of the defoliant should be [11]. Trials where fungicides were applied using VRA are listed in Table 4 . Reductions in herbicide use between $20 \%$ and $47 \%$ were reported.

In this paper, we assumed that an average reduction of $38 \%$ of herbicide use can be achieved (Table 4). There is the additional cost of obtaining biomass maps and some extra decision support. We assume that this is done using a Yara N-Sensor or GreenSeeker sensors on SensiSpray or conventional sprayers [11,53]. Sensor cost is (as above) 25 euro ha $^{-1}$. A regular pesticide sprayer can be used so there is no additional cost for precision application equipment. Herbicide products applied were Reglone, Spotlight, and Finale. The baseline cost of the herbicides is $68 € \mathrm{ha}^{-1}$ and the savings are $0.38 \times 68=26 € \mathrm{ha}^{-1}$. We do not assume an effect on the yield of the crop. 
Table 1. Soil herbicide trials in potato.

\begin{tabular}{|c|c|c|c|c|c|c|c|c|c|c|c|c|c|c|c|c|}
\hline Trial & Year & Location & Type & Crop & Herbicide & $\begin{array}{c}\text { Active } \\
\text { Ingredient }\end{array}$ & Data & Sensor & $\begin{array}{l}\text { Scan } \\
\text { Res. }\end{array}$ & $\begin{array}{l}\text { Sprayer } \\
\text { Width }\end{array}$ & $\begin{array}{c}\text { Reference } \\
\text { Amount }\end{array}$ & $\begin{array}{l}\text { Reference } \\
\text { a.i. }\end{array}$ & $\begin{array}{c}\text { VRA } \\
\text { Amount }\end{array}$ & VRA a.i. & $\begin{array}{l}\text { VRA Rel. } \\
\text { Amount }\end{array}$ & Reference \\
\hline & & & & & & & & & (m) & (m) & $\begin{array}{l}\left(\mathrm{L} \mathrm{ha}^{-1} \text { or }\right. \\
\left.\mathrm{kg} \mathrm{ha}^{-1}\right)\end{array}$ & $\begin{array}{c}\text { (g ai per } \\
\text { ha) }\end{array}$ & $\begin{array}{l}\left(\mathrm{L} \mathrm{ha}^{-1} \text { or }\right. \\
\left.\mathrm{kg} \mathrm{ha}^{-1}\right)\end{array}$ & $\begin{array}{c}\text { (g ai per } \\
\text { ha) }\end{array}$ & $\begin{array}{l}\text { (L or kg } \\
\text { per ha) }\end{array}$ & \\
\hline 1 & 2012 & $\begin{array}{c}\text { Biddinghuizen } \\
\left(52^{\circ} 29^{\prime} 4^{\prime \prime} \mathrm{N},\right. \\
\left.05^{\circ} 45^{\prime} 59^{\prime \prime} \mathrm{E}\right)\end{array}$ & Farm & Potato & Boxer & $\begin{array}{l}\text { Prosulfocarb } \\
\quad 800\end{array}$ & VRA map & $\gamma$-ray & 6 & 45 & 4.5 & 3600 & 3.5 & 2800 & 0.78 & [59] \\
\hline 2 & 2015 & $\begin{array}{l}\text { Vierhuizen } \\
\left(53^{\circ} 21^{\prime} 37^{\prime \prime} \mathrm{N},\right. \\
\left.06^{\circ} 17^{\prime} 28^{\prime \prime} \mathrm{E}\right)\end{array}$ & Farm & Onion & Stomp & $\begin{array}{l}\text { Pendimethalin } \\
\quad 400\end{array}$ & VRA map & Veris & 6 & 45 & 1.2 & 480 & 0.8 & 320 & 0.67 & [60] \\
\hline 3 & 2015 & $\begin{array}{l}\text { Huldenberg } \\
\left(51^{\circ} 44^{\prime} 10^{\prime \prime} \mathrm{N},\right. \\
\left.05^{\circ} 16^{\prime} 44^{\prime \prime} \mathrm{E}\right)\end{array}$ & Farm & Potato & Afalon & Linuron 450 & VRA map & Veris & 6 & 39 & 1.4 & 630 & 1.05 & 473 & 0.75 & $\begin{array}{l}\text { C. Kempenaar, } \\
\text { unpublished data. }\end{array}$ \\
\hline 4 & 2016 & $\begin{array}{l}\text { Huldenberg } \\
\left(51^{\circ} 44^{\prime} 10^{\prime \prime} \mathrm{N},\right. \\
\left.05^{\circ} 16^{\prime} 44^{\prime \prime} \mathrm{E}\right)\end{array}$ & Farm & $\begin{array}{l}\text { Winter } \\
\text { wheat }\end{array}$ & Liberator & $\begin{array}{l}\text { diflufenican } \\
100 \text { and } \\
\text { flufenacet } 400\end{array}$ & VRA map & Veris & 6 & 39 & 0.6 & 300 & 0.36 & 180 & 0.60 & $\begin{array}{l}\text { C. Kempenaar, } \\
\text { unpublished data. }\end{array}$ \\
\hline 5 & 2016 & $\begin{array}{c}\text { Abbenes } \\
\left(52^{\circ} 13^{\prime} 34^{\prime \prime} \mathrm{N},\right. \\
\left.04^{\circ} 37^{\prime} 09^{\prime \prime} \mathrm{E}\right)\end{array}$ & Farm & Potato & Boxer & $\begin{array}{l}\text { Prosulfocarb } \\
\quad 800\end{array}$ & VRA map & Veris & 6 & 39 & 4.5 & 3600 & 3.6 & 2880 & 0.80 & $\begin{array}{l}\text { C. Kempenaar, } \\
\text { unpublished data. }\end{array}$ \\
\hline 6 & 2017 & $\begin{array}{c}\text { Abbenes } \\
\left(52^{\circ} 13^{\prime} 34^{\prime \prime} \mathrm{N},\right. \\
\left.04^{\circ} 37^{\prime} 09^{\prime \prime} \mathrm{E}\right)\end{array}$ & Farm & Potato & Boxer & $\begin{array}{l}\text { Prosulfocarb } \\
\quad 800\end{array}$ & VRA map & Veris & 6 & 39 & 4.5 & 3600 & 3.7 & 2960 & 0.80 & $\begin{array}{c}\text { C. Kempenaar, } \\
\text { unpublished data. }\end{array}$ \\
\hline
\end{tabular}


Table 2. Late blight trials in potato.

\begin{tabular}{|c|c|c|c|c|c|c|c|c|c|c|c|c|c|c|c|c|}
\hline Trial & Years & Location & Fields & Type & Crop & Fungicide & $\begin{array}{c}\text { Active } \\
\text { Ingredient }\end{array}$ & $\begin{array}{c}\text { Reference } \\
\text { Amount }\end{array}$ & a.i. & $\begin{array}{c}\text { Scan } \\
\text { Resolution }\end{array}$ & Sensor & $\begin{array}{l}\text { Sprayer } \\
\text { Width }\end{array}$ & VRA & $\begin{array}{l}\text { VRA } \\
\text { a.i. }\end{array}$ & $\begin{array}{c}\text { VRA } \\
\text { Relative } \\
\text { Amount }\end{array}$ & Reference \\
\hline & & & & & & & & $\begin{array}{l}\left(\mathrm{L} \mathrm{ha}^{-1} \text { or }\right. \\
\left.\mathrm{kg} \mathrm{ha}^{-1}\right)\end{array}$ & & (m) & & (m) & $\begin{array}{l}\left(\mathrm{L} \mathrm{ha}^{-1} \text { or }\right. \\
\left.\mathrm{kg} \mathrm{ha}^{-1}\right)\end{array}$ & $\begin{array}{c}\text { (g ai } \\
\text { per ha) }\end{array}$ & & \\
\hline 1 & 2008-2009 & $\begin{array}{c}\text { Lelystad } \\
\left(52^{\circ} 32^{\prime} 28^{\prime \prime} \mathrm{N},\right. \\
\left.5^{\circ} 34^{\prime} 17^{\prime \prime} \mathrm{E}\right)\end{array}$ & 1 & Farm & Potato & Shirlan & fluazinam 500 & 0.4 & 200 & 10 & GreenSeeker & 27 & 0.31 & 154 & 0.77 & [53] \\
\hline 2 & 2008-2009 & $\begin{array}{c}\text { Lelystad } \\
\left(52^{\circ} 32^{\prime} 28^{\prime \prime} \mathrm{N},\right. \\
\left.5^{\circ} 34^{\prime} 17^{\prime \prime} \mathrm{E}\right)\end{array}$ & 1 & Farm & Potato & Infinito & $\begin{array}{c}\text { flupicolide } \\
62.5 \\
\text { propamocarb- } \\
\text { hydrochloride } \\
625 \\
\end{array}$ & 1.6 & 1.1 & 10 & GreenSeeker & 27 & 1.23 & 0.85 & 0.77 & [53] \\
\hline 3 & 2011 & $\begin{array}{c}\text { Lelystad } \\
\left(52^{\circ} 32^{\prime} 23^{\prime \prime} \mathrm{N},\right. \\
\left.5^{\circ} 33^{\prime} 44^{\prime \prime} \mathrm{E}\right)\end{array}$ & 1 & Farm & Potato & Shirlan & fluazinam 500 & 0.4 & 200 & 10 & GreenSeeker & 27 & 0.32 & 158 & 0.79 & [61] \\
\hline 4 & 2012-2015 & $\begin{array}{l}\text { Valthermond } \\
\left(52^{\circ} 52^{\prime} 25^{\prime \prime} \mathrm{N},\right. \\
\left.06^{\circ} 56^{\prime} 32^{\prime \prime} \mathrm{E}\right)\end{array}$ & 4 & Farm & Potato & Revus & $\begin{array}{l}\text { mandipropamid } \\
250\end{array}$ & 0.6 & 150 & 10 & $\mathrm{~N}$-Sensor & 39 & 0.47 & 119 & 0.79 & {$[60,62]$} \\
\hline
\end{tabular}

Table 3. Nitrogen sidedress trials in potato. Additional information about these trials has been published elsewhere $[57,63]$.

\begin{tabular}{|c|c|c|c|c|c|c|c|c|c|c|c|c|c|}
\hline \multirow[t]{2}{*}{ Year } & \multirow[t]{2}{*}{ Location } & \multirow[t]{2}{*}{ Soil } & \multirow[t]{2}{*}{ Type } & \multirow[t]{2}{*}{ Cultivar } & \multirow[t]{2}{*}{ Use Type } & \multirow[t]{2}{*}{ Sensor } & \multirow[t]{2}{*}{$\mathrm{N}_{\mathrm{opt}}$} & \multicolumn{2}{|c|}{ N Applied } & \multicolumn{2}{|c|}{ Tuber Yield } & \multirow[t]{2}{*}{ Reduction N Used } & \multirow[t]{2}{*}{ Reference. } \\
\hline & & & & & & & & Reference & Sidedress & Reference & Sidedress & & \\
\hline & & & & & & & $\left(\mathrm{kg} \mathrm{ha}^{-1}\right)$ & $\left(\mathrm{kg} \mathrm{ha}^{-1}\right)$ & $\left(\mathrm{kg} \mathrm{ha}^{-1}\right)$ & $\left(t_{h a}{ }^{-1}\right)$ & $\left(\mathrm{t} \mathrm{kg} \mathrm{ha}^{-1}\right)$ & $\left(\mathrm{kg} \mathrm{ha}^{-1}\right)$ & \\
\hline $1997-2000$ & Wageningen $\left(51^{\circ} 59^{\prime} 20^{\prime \prime} \mathrm{N}, 5^{\circ} 39^{\prime} 40^{\prime \prime} \mathrm{E}\right)$ & Sand & Experiment & Bintie & Ware & Cropscan & & & & & & $25-40$ & {$[56,64]$} \\
\hline 2002-2003 & Rolde $\left(52^{\circ} 57^{\prime} 55^{\prime \prime} \mathrm{N}, 06^{\circ} 39^{\prime} 5^{\prime \prime} \mathrm{E}\right)$ & Sand & Experiment & Seresta & Starch & Cropscan & & $220-250$ & $180-185$ & & & $40-65$ & [65] \\
\hline $2002-2003$ & Rolde $\left(52^{\circ} 57^{\prime} 55^{\prime \prime} \mathrm{N}, 06^{\circ} 39^{\prime} 25^{\prime \prime} \mathrm{E}\right)$ & Sand & Experiment & Mercator & Starch & Cropscan & & 155-185 & $140-160$ & & & $15-25$ & [65] \\
\hline $2002-2003$ & Colijnsplaat $\left(51^{\circ} 35^{\prime} 20^{\prime \prime} \mathrm{N}, 03^{\circ} 05^{\prime} 50^{\prime \prime} \mathrm{E}\right)$ & Loam & Experiment & Agria & Starch & Cropscan & & & $80-162$ & & & & {$[66,67]$} \\
\hline 2002-2003 & Colijnsplaat $\left(51^{\circ} 35^{\prime} 20^{\prime \prime} \mathrm{N}, 03^{\circ} 05^{\prime} 50^{\prime \prime} \mathrm{E}\right)$ & Loam & Experiment & Felsina & Starch & Cropscan & & & $131-176$ & & & & {$[66,67]$} \\
\hline 2010 & Valthermond $\left(52^{\circ} 52^{\prime} 25^{\prime \prime} \mathrm{N}, 06^{\circ} 56^{\prime} 32^{\prime \prime} \mathrm{E}\right)$ & Sand & Experiment & Seresta & Starch & Cropscan & 170 & 225 & $175+50$ & 47.8 & 48.5 & 0 & [68] \\
\hline 2010 & $\begin{array}{l}\text { Colijnsplaat }\left(51^{\circ} 35^{\prime} 20^{\prime \prime} \mathrm{N}, 03^{\circ} 05^{\prime} 50^{\prime \prime} \mathrm{E}\right) \\
\end{array}$ & Clay & Experiment & Victoria & Ware & Cropscan & 185 & 250 & $150+50$ & 44.4 & 46.8 & 50 & [68] \\
\hline 2011 & Valthermond $\left(52^{\circ} 52^{\prime} 25^{\prime \prime} \mathrm{N}, 06^{\circ} 56^{\prime} 32^{\prime \prime} \mathrm{E}\right)$ & Sand & Experiment & Merano & Starch & Cropscan & & $120+60$ & $120+45$ & 46.6 & 49.1 & 15 & [69] \\
\hline 2011 & Reusel $\left(51^{1} 19^{\prime} 16^{\prime \prime} \mathrm{N}, 05^{\circ} 10^{\prime} 35^{\prime \prime} \mathrm{E}\right)$ & Sand & Farm & Fontane & Ware & Cropscan & & 165 & $219+54$ & 78.6 & 87.2 & 0 & [69] \\
\hline 2011 & Reusel $\left(51^{\circ} 19^{\prime} 16^{\prime \prime} \mathrm{N}, 05^{\circ} 10^{\prime} 35^{\prime \prime} \mathrm{E}\right)$ & Sand & Farm & Fontane & Ware & Cropscan & & 229 & $229+27$ & 91.4 & 100.9 & 0 & [69] \\
\hline 2011 & Biddinghuizen $\left(52^{\circ} 29^{\prime} 04^{\prime \prime} \mathrm{N}, 05^{\circ} 45^{\prime} 59^{\prime \prime} \mathrm{E}\right)$ & Clay & Farm & Milva & Ware & N-Sensor & & $150+50$ & $150+50$ & 70.9 & 71 & 0 & [69] \\
\hline
\end{tabular}


Table 4. Potato haulm killing trials.

\begin{tabular}{|c|c|c|c|c|c|c|c|c|c|c|c|c|c|c|c|c|}
\hline $\begin{array}{l}\text { Trial } \\
\end{array}$ & Nr Fields & $\begin{array}{l}\text { Year(s) } \\
\end{array}$ & Location & Type & Herbicide & Active Ingredient & Method & $\begin{array}{l}\text { Sensor } \\
\end{array}$ & Scan Res. & Sprayer Width & Reference & VRA & Rel. Use & $\begin{array}{l}\text { Reference } \\
\end{array}$ & VRA & Reference \\
\hline & & & & & & & & & (m) & (m) & 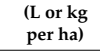 & $\begin{array}{l}\text { (L or kg } \\
\text { per ha) }\end{array}$ & (\%) & (g ai per ha) & (g ai per ha) & \\
\hline 1 & 11 & 2006-2008 & $\begin{array}{l}\text { Three farms in the province } \\
\text { of Flevoland, centered } \\
\text { around } 52^{\circ} 30^{\prime} 12^{\prime \prime} \mathrm{N} \\
5^{\circ} 37^{\prime} 42^{\prime \prime} \mathrm{E}\end{array}$ & Farm & Reglone & $\begin{array}{l}\text { diquat-dibromide } \\
200\end{array}$ & Nearby sensing & $N$-Sensor & 10 & 39 & 3.2 & 1.70 & $53 \%$ & 640 & 339 & [11] \\
\hline 2 & 12 & 2009-2011 & As above & Farm & Reglone & $\begin{array}{l}\text { diquat-dibromide } \\
200\end{array}$ & Nearby sensing & N-Sensor & 10 & 39 & 2.2 & 1.41 & $64 \%$ & 440 & 282 & [11] \\
\hline 3 & 4 & 2012-2013 & As above & Farm & Reglone & $\begin{array}{l}\text { diquat-dibromide } \\
200\end{array}$ & Nearby sensing & N-Sensor & 10 & 39 & 2.9 & 1.78 & $62 \%$ & 575 & 357 & [11] \\
\hline 4 & 6 & 2007-2011 &  & Farm & Reglone & $\begin{array}{c}\text { diquat-dibromide } \\
201\end{array}$ & Nearby sensing & $\begin{array}{l}\text { Greenseeker } \\
\text { Sensispray }\end{array}$ & 10 & 39 & 2.3 & 1.55 & $67 \%$ & 463 & 310 & [11] \\
\hline 5 & 2 & 2011-2012 & $\begin{array}{c}\text { Biddinghuizen }\left(52^{\circ} 29^{\prime} 04^{\prime \prime} \mathrm{N} \text {, }\right. \\
\left.05^{\circ} 45^{\prime} 59^{\prime \prime} \mathrm{E}\right)\end{array}$ & Farm & Reglone & $\begin{array}{l}\text { diquat-dibromide } \\
202\end{array}$ & Remote & UAV + Multispec & 0.5 & 39 & 2.3 & 1.40 & $62 \%$ & 450 & 279 & [11] \\
\hline 6 & 1 & 2011 & As above & Farm & Reglone & $\begin{array}{l}\text { diquat-dibromide } \\
203\end{array}$ & Remote sensing & $\begin{array}{l}\text { Satellite- based } \\
\text { (Worldview-2) }\end{array}$ & 2 & 39 & 3 & 2.4 & $80 \%$ & 600 & 480 & [11] \\
\hline 7 & 1 & 2013 & $\begin{array}{l}\text { Valthermond (52.552 } 25^{\prime \prime} \mathrm{N} \text {, } \\
\left.06^{\circ} 56^{\prime} 32^{\prime \prime} \mathrm{E}\right)\end{array}$ & Farm & Reglone & $\begin{array}{c}\text { diquat-dibromide } \\
204\end{array}$ & Remote sensing & $\begin{array}{c}\text { Satellite- based } \\
\text { Formosat }\end{array}$ & 8 & 39 & 3 & 2.19 & $73 \%$ & 600 & 438 & [11] \\
\hline 8 & 1 & 2014 & $\begin{array}{l}\text { Zijen }\left(53^{\circ} 0^{\prime 2} 5^{\prime \prime} \mathrm{N},\right. \\
\left.06^{\circ} 32^{4} 47^{\prime \prime} \mathrm{E}\right)\end{array}$ & Farm & Reglone & $\begin{array}{l}\text { diquat-dibromide } \\
205\end{array}$ & Nearby sensing & $N$-Sensor & 10 & 39 & 3 & 2.13 & $71 \%$ & 600 & 426 & [60] \\
\hline 9 & 1 & 2014 & $\begin{array}{l}\text { Valthermond (52 } 52^{\circ} 52^{\prime} 25^{\prime \prime} \mathrm{N}, \\
\left.06^{\circ} 56^{\prime} 32^{\prime \prime} \mathrm{E}\right)\end{array}$ & Farm & Reglone & $\begin{array}{c}\text { diquat-dibromide } \\
206\end{array}$ & Nearby sensing & N-Sensor & 10 & 39 & 3 & 1.83 & $61 \%$ & 600 & 366 & [60] \\
\hline 10 & 1 & 2016 & Ell $51^{\circ} 12^{\prime} 17^{\prime \prime} \mathrm{N}, 5^{\circ} 48^{\prime} 23^{\prime \prime} \mathrm{E}$ & Farm & Reglone & $\begin{array}{l}\text { diquat-dibromide } \\
207\end{array}$ & Nearby sensing & $N$-Sensor & 10 & 39 & 3 & 1.68 & $56 \%$ & 600 & 336 & $\begin{array}{c}\text { C. } \\
\text { Kempenaar, } \\
\text { unpublished } \\
\text { data } \\
\end{array}$ \\
\hline 11 & 1 & 2016 & $\begin{array}{c}\text { Abbeneses }\left(52^{\circ} 13^{3} 34^{\prime \prime} \mathrm{N}\right. \\
\left.04^{\circ} 37^{\prime} 09^{\prime \prime} \mathrm{E}\right)\end{array}$ & Farm & $\begin{array}{c}\text { Spotlight } \\
\text { Plus }\end{array}$ & $\begin{array}{l}\text { carfentrazone-ethyl } \\
60\end{array}$ & Remote sensing & Satellite-based & & 39 & 1 & 0.59 & $59 \%$ & 60 & 35.4 & $\begin{array}{c}\text { C. } \\
\text { Kempenaar, } \\
\text { unpublished } \\
\text { data } \\
\end{array}$ \\
\hline
\end{tabular}




\subsubsection{Four Techniques Combined}

A truly precision-agriculture minded farmer will apply all four above-mentioned techniques. In fact, once a person has made the step to use one precision agriculture technique, applying a second technique will be easier because the data processing tools will already be set up. There will also be a benefit of scale because three of the four techniques are based on a canopy reflectance map of the crop. Above we have assumed that an $N$-Sensor is used for this measurement. However, if canopy reflectance maps are needed throughout the season, it makes sense to subscribe to the vegetation monitoring system of Bioscope (http:/ / www.projectbioscope.eu/) which is based on the integrated use of satellites (cheap, but in The Netherlands often not available due to clouds) and Unmanned Aerial Vehicles. We assume that at a cost of $70 € \mathrm{ha}^{-1} \mathrm{y}^{-1}$ a subscription is available that delivers canopy reflectance images at 10-day intervals through the entire growing season.

\subsection{Olives in Greece}

Olive (Olea europaea L.) is a major crop in Greece. In 2014, olives were grown on 938,270 ha and the production of olive oil was $1,780,560$ tonnes [70]. The olive production sector is characterized by a large number of small operators and a large variation in production levels. We focused on an orchard with a high level of productivity which was supported by a high level of inputs. In such an orchard it is especially attractive to apply variable rate application of fertilizer.

Variable rate application was developed in an olive oil tree plantation (9.1 ha, 181 trees ha $\left.{ }^{-1}\right)$ in Southern Greece with a marked spatial variability of soil properties (Figure 1). In 2008 and 2009, site-specific management was carried out for $\mathrm{P}, \mathrm{K}$, and lime after soil sampling at a 10 samples per ha sampling strategy [71]. The field was sectioned according to the delineated management zones and the generated contours. For P and K, only two management zones were created to be more applicable for farming practices. For soil $\mathrm{P}$ the threshold was set to $45 \mathrm{ppm}$ and for $\mathrm{K}$ it was $350 \mathrm{ppm}$ [71]. For the $\mathrm{P}$ application, in the low-P management zone, $1 \mathrm{~kg}$ fertilizer per tree was applied. No $\mathrm{P}$ fertilizer was applied for the high-P management zone. The farmer's practice was $1 \mathrm{~kg}$ of $\mathrm{P}$ per tree. For $\mathrm{K}$ application, in the low-K management zone, $2 \mathrm{~kg} \mathrm{~K}$ fertilizer per tree was applied, whereas $1 \mathrm{~kg} \mathrm{~K}$ fertilizer per tree was applied in the high-K zone. The farmer's practice was to apply $2 \mathrm{~kg}$ of $\mathrm{K}$ per tree. For $\mathrm{pH}$, lime was applied at a rate of $5 \mathrm{~kg}$ per tree to the areas in the field in which $\mathrm{pH}$ was less than 6.5. The farmer's practice was to apply $5 \mathrm{~kg}$ per tree in all trees every four years.

Fertilizer application rates according to the farmer's practice and under VRA are summarized in Table 5. No significant differences in yield were seen between the different management zones. In the 13 positions out of 91 where $\mathrm{pH}$ was below 6.5 and lime was applied, mean $\mathrm{pH}$ increased from 5.9 to 7.0. Overall, it was concluded that the chosen algorithms for variable rate application of $\mathrm{P}, \mathrm{K}$, and lime allowed a reduction in input use to be achieved and avoided a negative impact on productivity. 


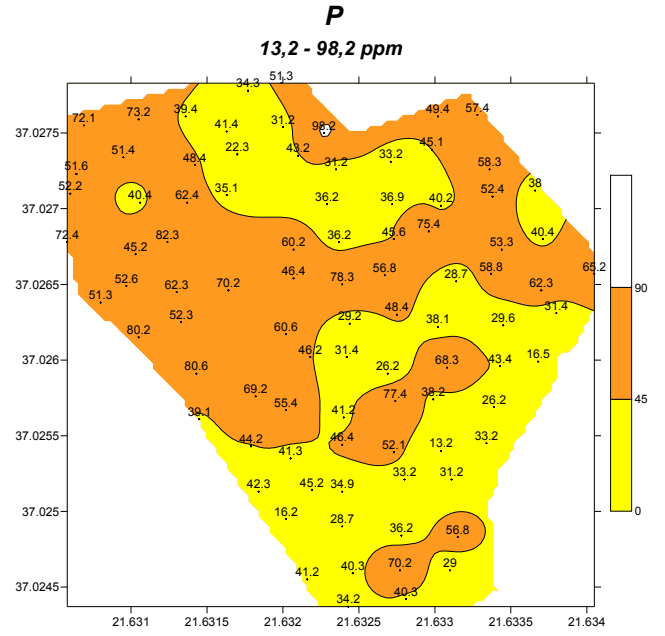

(a)

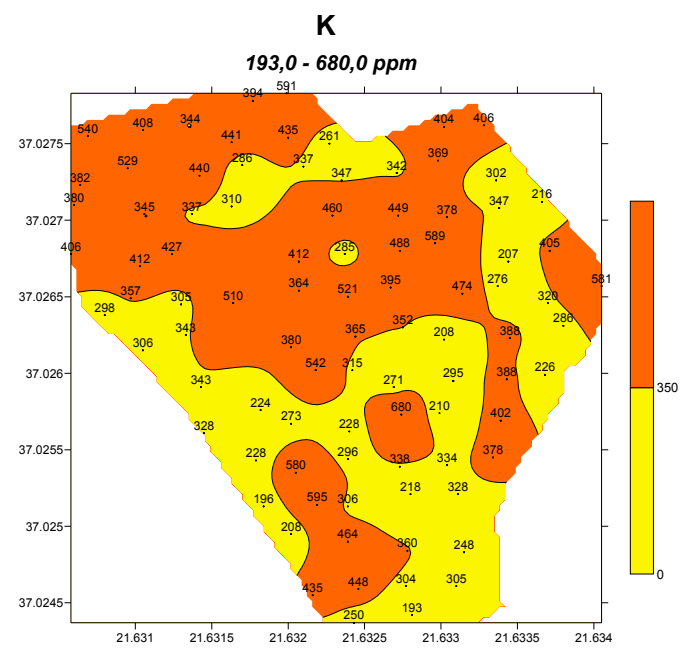

(b)

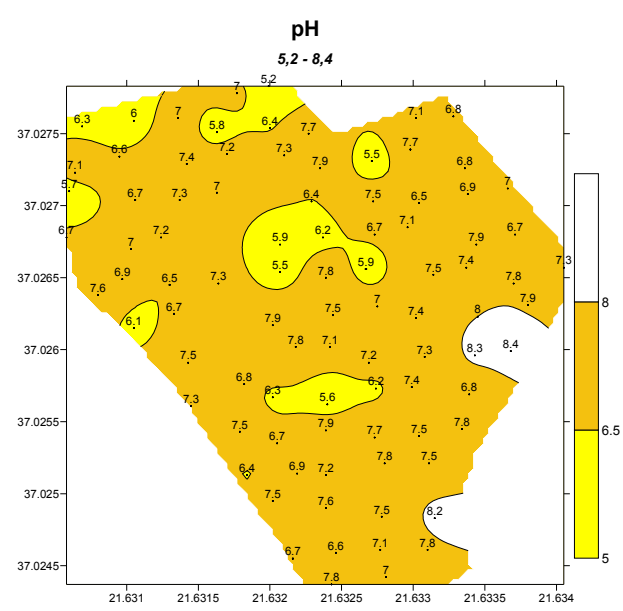

(c)

Figure 1. Delineated management zones in olives (a) for $\mathrm{P}$, using a $45 \mathrm{ppm}$ threshold; (b) for $\mathrm{K}$, using a 350 ppm threshold; (c) Interpolated map of soil $\mathrm{pH}$.

Table 5. Olive VRA trials.

\begin{tabular}{cccccrc}
\hline Year & Location & Application & Farmer's Practice kg Fertilizer & VRA Application kg Fertilizer & VRA Relative Amount & Reference \\
\hline \multirow{2}{*}{$2007-2008$} & $\begin{array}{c}\text { Greece } \\
\left(37^{\circ} 01^{\prime} 35^{\prime \prime} \mathrm{N},\right. \\
\left.21^{\circ} 37^{\prime} 55^{\prime \prime} \mathrm{E}\right)\end{array}$ & $\mathrm{P}$ & $91 \times 1 \mathrm{~kg}=91 \mathrm{~kg}$ & $37 \times 1 \mathrm{~kg}=37 \mathrm{~kg}$ & 0.41 & {$[71]$} \\
\hline & $\mathrm{K}$ & $91 \times 2 \mathrm{~kg}=182 \mathrm{~kg}$ & $34 \times 2 \mathrm{~kg}+57 \times 1 \mathrm{~kg}=125 \mathrm{~kg}$ & 0.69 & {$[71]$} \\
\hline & Lime & $91 \times 5 \mathrm{~kg}=455 \mathrm{~kg}$ & $13 \times 5 \mathrm{~kg}=65 \mathrm{~kg}$ & 0.14 & {$[71]$} \\
\hline
\end{tabular}

The calculations for profit and social profit were based on a published LCA analysis [72] but some numbers were adjusted to match the current experiment. The productivity of olives was set $8500 \mathrm{~kg} \mathrm{ha}^{-1}$, which is the average value in the two years of the above-mentioned experiment. The productivity of olive oil was $1700 \mathrm{~kg} \mathrm{ha}^{-1}$. Dimethoate $\left(1.8 \mathrm{~kg} \mathrm{ha}^{-1}\right)$ was used to control olive fly. The standard tariff of the oil mill is $12 \%$ of the value of the oil. The average cost of renting agricultural land in Greece is $400 € \mathrm{ha}^{-1}$. The cost of machines (depreciation, maintenance, insurance) was calculated to be $320 € \mathrm{ha}^{-1}$, based on data of the farmer in the above experiment. Each tree is irrigated with $8000 \mathrm{~kg}$ of water per year but there is no charge for water use.

Variable rate application of nutrients and lime is done on the basis of information about the spatial variability of soil conditions. This information is derived from soil samples. In the experiment described 10 samples per ha were taken but it is likely that in commercial practice fewer samples will 
be taken. At five samples per ha, taken once every four years, and at a price of 50 euro per sample, the total cost of this sampling scheme is $62.5 € \mathrm{ha}^{-1} \mathrm{y}^{-1}$.

\section{Results}

Results for potato are shown in Table 6 and Table S1. Emission of GHG for potatoes according to our calculations is $4329 \mathrm{~kg} \mathrm{CO}_{2}$-eq for a production of $53,560 \mathrm{~kg}$ of potatoes, which is $83 \mathrm{~kg} \mathrm{t}^{-1}$; this is comparable with the $77 \mathrm{~kg} \mathrm{t}^{-1}$ reported elsewhere [73].

The externality pesticide toxicity is reduced most by using variable rate application of fungicides for late blight control: this reduces the environmental cost from $82 € \mathrm{ha}^{-1}$ to $73 € \mathrm{ha}^{-1}$ (an $11 \%$ reduction; see Table 7). VRA for soil herbicides (SH) reduces this externality from $82 € \mathrm{ha}^{-1}$ to $76 €$ $\mathrm{ha}^{-1}$ (a $7 \%$ reduction) and using variable rate application of potato haulm killing herbicides (PHK) reduces this externality from $82 € \mathrm{ha}^{-1}$ to $77 € \mathrm{ha}^{-1}$ (5\% reduction). Using variable rate application for all three (SH, PHK, and LB) has of course the largest effect: it reduces the externality pesticide toxicity from $82 € \mathrm{ha}^{-1}$ to $63 € \mathrm{ha}^{-1}$ ( $23 \%$ reduction).

The externality GHG emissions is reduced by variable rate application for sidedress $\mathrm{N}(\mathrm{SN})$ from $136 € \mathrm{ha}^{-1}$ to $124 € \mathrm{ha}^{-1}$ (a 9\% reduction; see Table 7). VRA for SN also reduces eutrophication by $17 \%$.

Profit is increased by variable rate application of herbicides (11\%) but the other PA techniques have just a small or even a slightly negative effect on profit. When all four PA techniques are applied together, profit is increased by $13 \%$.

Social profit is affected in the same way as profit, but the effects are slightly larger because the increase in profit and the decrease in environmental cost operate in the same direction. Thus, as shown in Table 7 , social profit is increased by $13 \%$ if VRA for $\mathrm{SH}$ is used and by $26 \%$ when all four techniques are used together. Profit, externalities and social profit summarized in Figure 2.

Results for olive are detailed in Table 8 and Table S2 and summarized in Figure 3. PA in olives results in substantial reductions in $\mathrm{P}, \mathrm{K}$, and lime use. Fertilizer is relatively cheap, however, and the soil samples needed for variable rate application are relatively expensive, which when taken together means that profit is increased only slightly (compare $\mathrm{SN}$ in potatoes). Likewise, the impact on social profit is small. 
Table 6. Gross margin and social profit of potatoes under standard practice. In Table S1 full details are given for this practice, for the four PA technologies, and for the four PA technologies combined.

\begin{tabular}{|c|c|c|c|c|c|c|c|c|c|c|c|}
\hline & & & STANDARI & ACTIC & & & & & & & \\
\hline & & & Quantity & & & & Value & & & & \\
\hline Item & Active Ingredient & Units & $\begin{array}{c}\text { Input } \\
\text { (Units/ha) }\end{array}$ & $\begin{array}{l}\text { EIQ } \\
\text { (/ha) }\end{array}$ & $\begin{array}{l}\mathrm{CO}_{2} \text {-eq } \\
(\mathrm{kg} / \mathrm{ha})\end{array}$ & $\begin{array}{c}\text { Nutr. Lost } \\
\text { (kg/ha) }\end{array}$ & $\begin{array}{l}\text { Input } \\
(€ / \text { ha) }\end{array}$ & $\begin{array}{c}\text { EIQ } \\
(€ / \mathrm{ha})\end{array}$ & $\begin{array}{c}\mathrm{CO}_{2} \text {-eq } \\
(\boldsymbol{\epsilon} / \mathrm{ha})\end{array}$ & $\begin{array}{l}\text { Biodiv } \\
\text { (€/ha) }\end{array}$ & $\begin{array}{c}\text { Eutroph. } \\
(\in / \text { ha })\end{array}$ \\
\hline $\begin{array}{l}\text { main product } \\
\text { Potatoes }\end{array}$ & & $\mathrm{kg}$ & 53,560 & & & & $€ 8332$ & & & & \\
\hline $\begin{array}{l}\text { planting material } \\
\text { seed potatoes }\end{array}$ & & $\mathrm{kg}$ & 2700 & & 273.4 & & $€ 756$ & & 68.61 & & \\
\hline $\begin{array}{c}\text { fertilizers } \\
\text { CAN } \\
\text { triple-super-phosphate } \\
\text { Potassium }\end{array}$ & & $\begin{array}{c}\mathrm{kg} \mathrm{N} \\
\mathrm{kg} \mathrm{P}_{2} \mathrm{O}_{5} \\
\mathrm{~kg} \mathrm{~K}_{2} \mathrm{O}\end{array}$ & $\begin{array}{l}252 \\
105 \\
180 \\
\end{array}$ & & $\begin{array}{c}2237.8 \\
58.8 \\
77.4\end{array}$ & $\begin{array}{c}50 \\
2\end{array}$ & $\begin{array}{l}€ 265 \\
€ 105 \\
€ 115\end{array}$ & & $\begin{array}{l}€ 70.44 \\
€ 1.85 \\
€ 2.44\end{array}$ & $\begin{array}{r}€ 3.33 \\
€ 15.12\end{array}$ & $\begin{array}{c}€ 7.80 \\
€ 14.78\end{array}$ \\
\hline $\begin{array}{c}\text { herbicide (pre-emergence) } \\
\text { Boxer } 80 \% \\
\end{array}$ & prosulfocarb (800) & $\mathrm{L}$ & 4 & 49.1 & 73.9 & & $€ 54$ & $€ 18$ & $€ 2.33$ & & \\
\hline $\begin{array}{c}\text { herbicide } \\
\text { (pre/post-emergence) } \\
\text { Sencor } 70 \%\end{array}$ & metribuzin $(70 \%)$ & $\mathrm{L}$ & 0.5 & 9.9 & 8.1 & & $€ 22$ & $€ 4$ & 60.25 & & \\
\hline $\begin{array}{l}\text { fungicide } \\
\text { Signum } \\
\text { Ranman } \\
\text { Infinito } \\
\text { Revus }\end{array}$ & $\begin{array}{c}\text { boscalid }(27 \%) \text {, and } \\
\text { pyraclostrobine }(7 \%) \\
\text { cyazofamid }(160) \\
\text { fluopicolide }(6.25 \%) \text {, and } \\
\text { propamocarb }(62.5 \%) \\
\text { mandipropamid }(250)\end{array}$ & $\begin{array}{l}\text { L } \\
\text { L } \\
\text { L } \\
\text { L } \\
\text { L } \\
\text { L }\end{array}$ & $\begin{array}{c}0.4 \\
3 \\
3 \\
4.8 \\
\\
3.6\end{array}$ & $\begin{array}{l}2.9 \\
0.8 \\
7.4 \\
7.8 \\
71.7 \\
24.4\end{array}$ & $\begin{array}{l}1.5 \\
0.4 \\
6.9 \\
4.3 \\
42.9 \\
12.9\end{array}$ & & $\begin{array}{c}€ 26 \\
€ 156 \\
€ 96 \\
€ 131\end{array}$ & $\begin{array}{c}€ 1 \\
€ 0 \\
€ 3 \\
€ 3 \\
€ 27 \\
€ 9\end{array}$ & $\begin{array}{l}€ 0.05 \\
€ 0.01 \\
€ 0.22 \\
€ 0.14 \\
€ 1.35 \\
€ 0.41\end{array}$ & & \\
\hline $\begin{array}{l}\text { insecticide } \\
\text { Calypso } \\
\text { Karate }\end{array}$ & $\begin{array}{l}\text { thiaclopryd (480) } \\
\text { lambda-cyhalothrin (100) }\end{array}$ & $\begin{array}{l}\mathrm{L} \\
\mathrm{L}\end{array}$ & $\begin{array}{l}0.15 \\
0.05\end{array}$ & $\begin{array}{l}2.3 \\
0.2\end{array}$ & $\begin{array}{l}1.3 \\
0.2\end{array}$ & & $\begin{array}{c}€ 26 \\
€ 6\end{array}$ & $\begin{array}{l}€ 1 \\
€ 0\end{array}$ & $\begin{array}{l}€ 0.04 \\
€ 0.01\end{array}$ & & \\
\hline $\begin{array}{l}\text { haulm killing (defoliant) } \\
\text { Reglone } 20 \% \\
\text { sprout suppressant } \\
\text { chloorprofam (300) }\end{array}$ & $\begin{array}{l}\text { diquat bromide (200) } \\
\text { chloorprofam (300) }\end{array}$ & $\begin{array}{l}\mathrm{L} \\
\mathrm{L}\end{array}$ & $\begin{array}{c}4 \\
1.6\end{array}$ & $\begin{array}{l}31.4 \\
14.7\end{array}$ & $\begin{array}{l}23.4 \\
11.1\end{array}$ & & $\begin{array}{l}€ 68 \\
€ 50\end{array}$ & $\begin{array}{l}€ 12 \\
€ 5\end{array}$ & $\begin{array}{l}60.74 \\
60.35\end{array}$ & & \\
\hline $\begin{array}{c}\text { energy } \\
\text { electricity potato storage } \\
\text { diesel fuel } \\
\end{array}$ & & $\begin{array}{c}\mathrm{kWh} \\
\mathrm{L}\end{array}$ & $\begin{array}{c}1071 \\
260\end{array}$ & & $\begin{array}{l}563.3 \\
842.4\end{array}$ & & $\begin{array}{l}€ 164 \\
€ 285\end{array}$ & & $\begin{array}{l}€ 17.73 \\
€ 26.52\end{array}$ & & \\
\hline $\begin{array}{l}\text { other costs } \\
\text { into storage/from storage } \\
\text { interest } \\
\text { soil sample for mineral N } \\
\text { potato pol }\end{array}$ & & & $\begin{array}{c}54 \\
€ 1,252 \\
0.1 \\
1\end{array}$ & & & & $\begin{array}{c}€ 96 \\
€ 69 \\
€ 4 \\
€ 19\end{array}$ & & & & \\
\hline
\end{tabular}


Table 6. Cont.

\begin{tabular}{|c|c|c|c|c|c|c|c|c|c|c|c|}
\hline \multicolumn{12}{|c|}{ STANDARD PRACTICE } \\
\hline & & & Quantity & & & & Value & & & & \\
\hline Item & Active Ingredient & Units & $\begin{array}{c}\text { Input } \\
\text { (Units/ha) }\end{array}$ & $\begin{array}{c}\text { EIQ } \\
\text { (/ha) }\end{array}$ & $\begin{array}{l}\mathrm{CO}_{2} \text {-eq } \\
(\mathrm{kg} / \mathrm{ha})\end{array}$ & $\begin{array}{c}\text { Nutr. Lost } \\
\text { (kg/ha) }\end{array}$ & $\begin{array}{l}\text { Input } \\
\text { (€/ha) }\end{array}$ & $\begin{array}{c}\text { EIQ } \\
\text { (€/ha) }\end{array}$ & $\begin{array}{c}\mathrm{CO}_{2} \text {-eq } \\
(\epsilon / \mathrm{ha})\end{array}$ & $\begin{array}{c}\text { Biodiv } \\
\text { (€/ha) }\end{array}$ & $\begin{array}{c}\text { Eutroph } \\
(€ / \text { ha) }\end{array}$ \\
\hline revenue & & & & & & & $€ 8332$ & & & & \\
\hline cost of inputs & & & & & & & $€ 2513$ & & & & \\
\hline revenue-inputs & & & & & & & $€ 5819$ & & & & \\
\hline $\begin{array}{c}\text { cost of labour and } \\
\text { machines }\end{array}$ & & & & & & & $€ 2777$ & & & & \\
\hline land rent & & & & & & & $€ 1000$ & & & & \\
\hline gross margin & & & & & & & $€ 2042$ & & & & \\
\hline $\begin{array}{l}\text { sum externalities } \\
\text { social profit }\end{array}$ & & & & 222 & 4240 & & & $€ 82$ & $€ 133$ & $€ 18$ & $\begin{array}{c}€ 23 \\
€ 1785\end{array}$ \\
\hline
\end{tabular}

Table 7. Percent change under PA scenarios in potato, relative to the base scenario of a conventional production system. The column headings mean the following: $\mathrm{EIQ}=$ Environment Impact Quotient; $\mathrm{CO}_{2}=$ Emission of $\mathrm{CO}_{2}$-eq; BIODIV = loss of biodiversity; EUTR = eutrophication; $P=$ profit; $S P=$ social profit.

\begin{tabular}{ccccccc}
\hline Case & EIQ & CO $_{\mathbf{2}}$ & BIODIV & EUTR & $\boldsymbol{P}$ & SP \\
\hline VRA Soil herbicides & -7 & -2 & 0 & 0 & 11 & 13 \\
VRA Late blight & -11 & 1 & 0 & 0 & 3 & 3 \\
VRA Sidedress N & 0 & -9 & -3 & -17 & 1 & 2 \\
VRA PHK & -5 & 0 & 0 & 0 & -1 & -1 \\
ALL & -23 & -9 & -3 & -17 & 21 & 26 \\
\hline
\end{tabular}




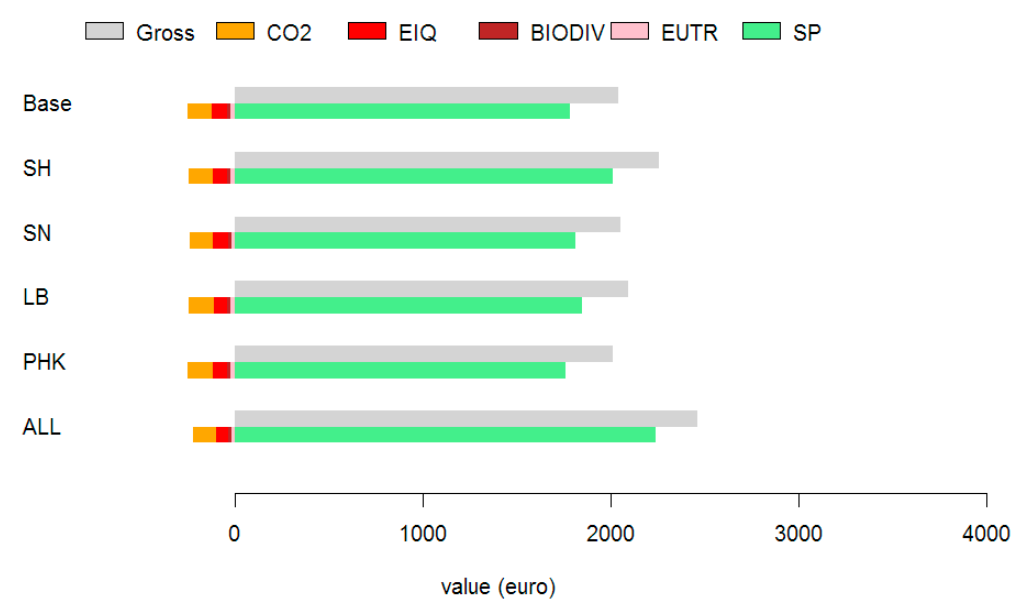

Figure 2. Social profit of potato production under several scenarios (Base = conventional practice; $\mathrm{SH}=$ variable rate application of soil herbicides; $\mathrm{SN}$ = variable rate application of sidedress $\mathrm{N}$; $\mathrm{LB}=$ variable rate application of late blight fungicide; $\mathrm{PHK}=$ variable rate application of potato haulm killing herbicide; $\mathrm{ALL}=$ all four variable rate scenarios combined). Gross = value of production minus costs excluding land and capital; $\mathrm{CO}_{2}=$ external cost of $\mathrm{CO}_{2}$ emission; $\mathrm{EIQ}=$ external cost of pesticide toxicity; BIODIV = external cost of loss of biodiversity due to the application of fertilizers; EUTR = external cost of eutrophication resulting from application of fertilizers; $S P=$ social profit (Gross-CO 2 -EIQ-BIODIV-EUTR-SP).

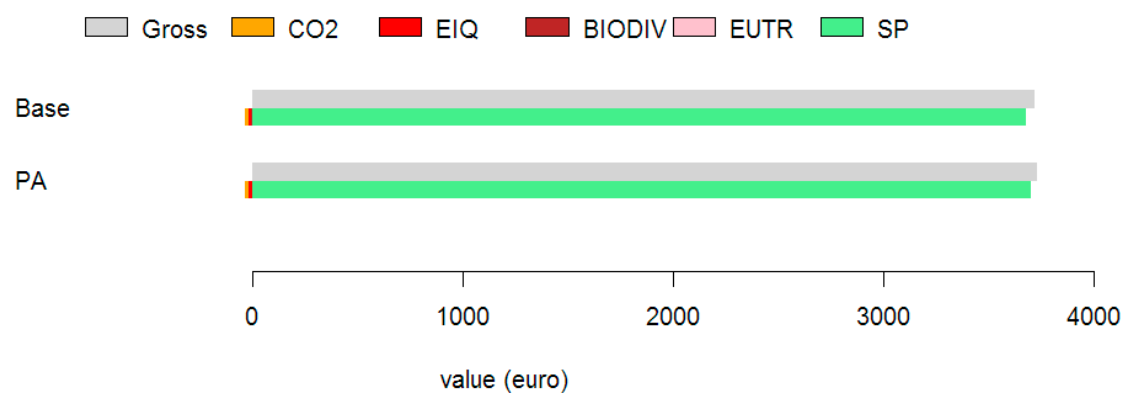

Figure 3. Social profit of olive production under conventional and precision agriculture. For explanations of legend, see Figure 2. 
Table 8. Gross margin and social profit of olive production under standard practice. In Table S2 full details are given for this practice and for the practice in which VRA of $P, K$, and lime is used.

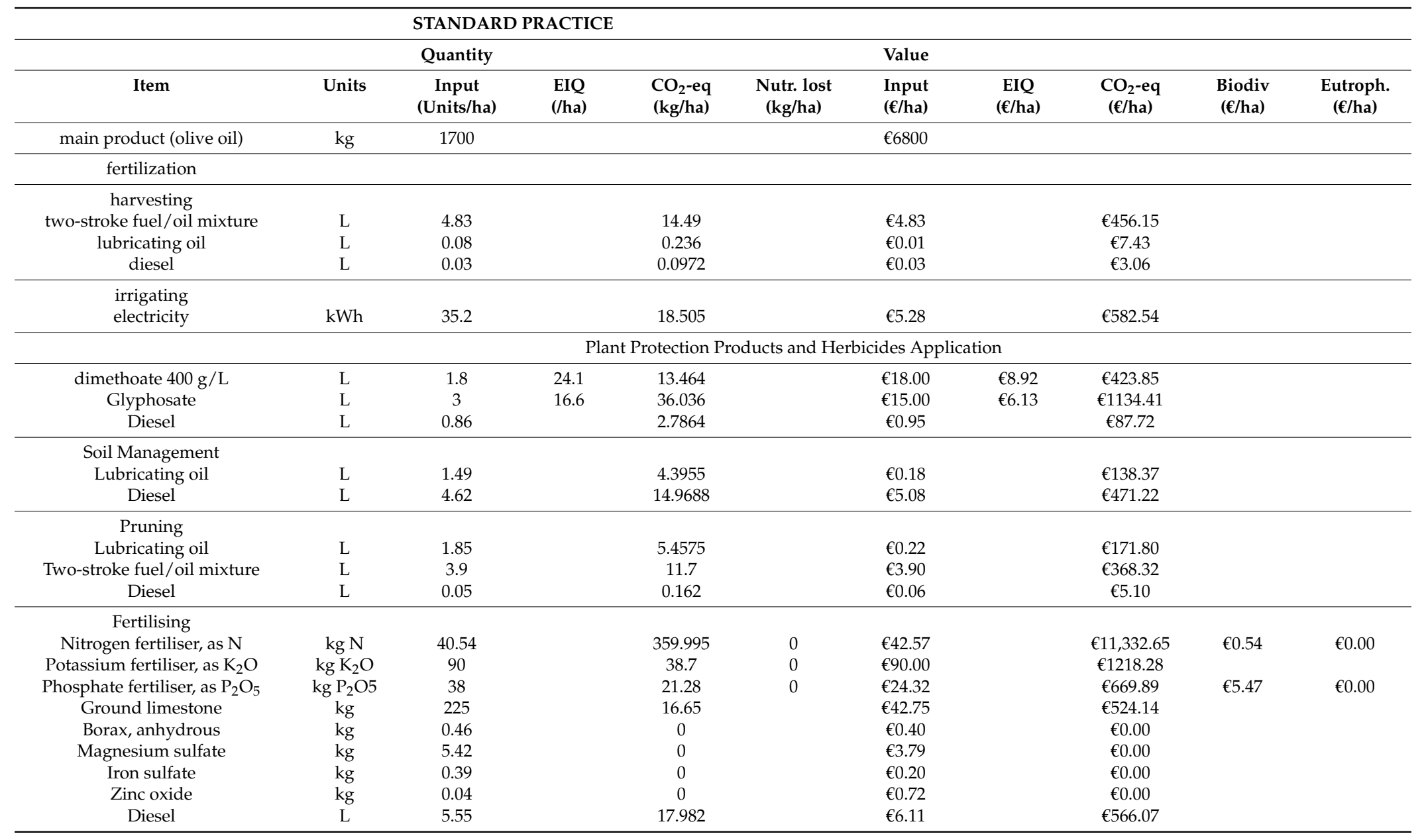


Table 8. Cont.

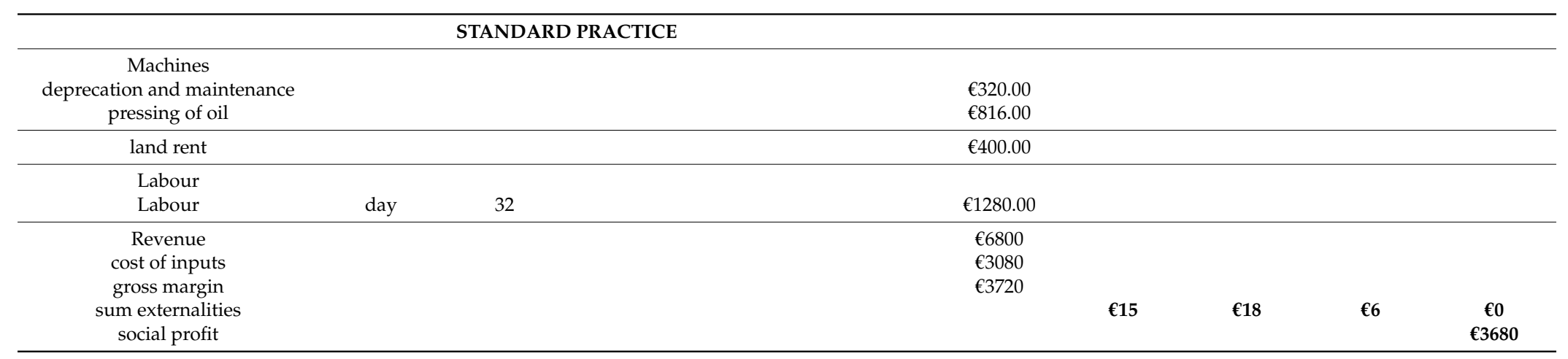




\section{Discussion}

Our results show that in potato production, PA can lead to a reduction in pesticide use of $23 \%$ (expressed as EIQ) and a reduction of nitrogen fertilizer use of $15 \%$. In olive production, the use of potassium fertilizer is reduced by $31 \%$, of phosphate fertilizer by $59 \%$, and of lime by $86 \%$. A significant reduction in environmental impact is associated with this reduction in input use.

Farmers incur some costs when they use PA but profitability increases nevertheless. The numbers are different for each PA technique. In the case of expensive inputs (some pesticides), reduction in input use results quickly in cost reduction, but in the case of less expensive inputs (nitrogen fertilizer), the cost savings are approximately equal to the extra cost to the farmer. In this case there is no or just a small economic benefit to the farmer. However, overall the conclusion is that PA increases profitability as well as sustainability.

Our quantification of the contribution of PA to sustainability started with a detailed listing of inputs, outputs, and externalities. Many methods to measure sustainability start with this step. This first step provides valuable insight into the effect of using PA but it leaves unanswered the kind of question that was posed in the Introduction section, namely do we increase sustainability when pesticide use is reduced by a certain amount and fuel use is increased by a certain other amount? In order to answer this and similar questions, ultimately sustainability must be quantified using hard measurements which can be subjected to rigorous analysis [74]. In this paper we attempt to progress towards such analysis by making explicit the trade-off between incompatible quantities. We use shadow prices to express all quantities using a common denominator. This enabled us to subtract both conventional costs and (external) environmental costs from revenue and thus calculate social profit as an aggregated measure of sustainability.

It can be argued that economic valuation of important environmental impacts such as loss of biodiversity and pollution of natural resources is not meaningful. However, it is clear that our society needs to do a better job of protecting intangible values and common resources while at the same time continuing to create economic value. Human beings are very familiar with making trade-offs and economic value plays a role, if often on a subconscious level. We argue that economic valuation can be a tool to help make decisions that take into account previously invisible goods and services such as biodiversity and other forms of natural capital.

A more serious drawback of our method is perhaps that we have not taken into account temporal effects: a full analysis of sustainability should include temporal effects [75]. In this paper we feel justified in leaving out temporal aspects because we are interested in relative sustainability. That is, we attempt to answer the question whether using PA is "more" sustainable than not using PA. We do not make a statement about whether either production system could be continued indefinitely $[19,76]$. If the goal is to make statements about longer time horizons, many more aspects need to be included in the analysis. For example, changes in rainfall patterns due to climate change are expected to increase nutrient leaching and eutrophication [77].

Farmers make decisions at the level of the farm. An important aspect is how different crops are grown in a rotation. Our analysis at the level of a crop of potatoes does not address all issues important to a farmer. However, potato is the most profitable crop in The Netherlands and profitability of the farm will be determined in large part by the performance of the potato crop. Olives are a permanent crop and then there is no rotation.

The size of a farm is an important determinant for some per-ha prices and therefore for the profitability of PA technologies. In earlier work it was calculated that an arable farm in the Netherlands must be $60-125$ ha or larger, depending on the crops grown, in order for the investments in PA technology to pay off [53], In this paper we assumed 100 ha of potatoes to calculate the price for some technologies. However, in potato production combining all four PA technologies we assumed that all sensor measurements are bought as a service at a fixed price per ha-then, the size of the farm does not matter. 
There is of course both uncertainty and variability of prices. For example, the price of potatoes of $0.16 € \mathrm{~kg}^{-1}$ will not be realized in all years; likewise, the prices of inputs may vary over time. Taken together, the profitability of an individual farm may differ from our calculations.

Our shadow prices for pollution are such that the effect on sustainability as measured with social profit is not very large. This gives rise to the feeling that there is a discrepancy between the intuitive importance of reducing pesticide use and the magnitude of the calculated effect on sustainability. This may be due at least in part to how we calculate the environmental cost of using pesticides. EIQ is probably the best measure available [78]. However, it provides an underestimation because it does not take into account the external costs associated with acute and chronic pesticide poisoning of humans, the long term effects on the environment, and synergetic and multiplicative effects of the use of pesticides. There is insufficient data available to take these mechanisms into account.

Some olive orchards in Greece may be located close to high-value natural areas. If so, the shadow prices that we used may underestimate the true cost.

Policy-makers are increasingly using economic valuation when making decisions and people are increasingly receptive to the idea that the costs and benefits of a production activity can be considered in monetary terms [79]. Nevertheless, a main weakness of economic valuation lies in the complexity of the methodologies and the modelling techniques. Also, the adoption of multiple assumptions often reduces credibility of the estimates. Finally, the limited knowledge about the environmental and socio-economic consequences caused by some externalities and about the time-spans at which they operate (e.g., future impacts of pesticides), results in the exclusion of external costs that cannot be accounted for in the valuation exercise.

Moreover, to which extent the future external costs should be discounted to estimate an optimal intergenerational shadow price, is still an issue which is currently subject to lively debate. This has not to do with the choice of the economic valuation method but with ethical considerations.

We have mentioned four PA technologies in potato production but additional technologies are in the pipeline. Variable rate planting of potatoes in response to soil texture is a promising technique [80] even though the mechanism through which it can increase yield is only partly understood [81] but there are indications that by applying a variable planting rate the yield of marketable tubers may be increased by $5 \%$. Also in the pipeline is that the resolution at which measurements and variable rate applications are made will increase and this will result in greater savings. Currently, measurements in potato production are typically made at $30 \times 30 \mathrm{~m}^{2}$ but with ever-decreasing cost of sensors and application technology, in the future a resolution of $1 \times 1 \mathrm{~m}^{2}$ seems within reach. It has been calculated that in the case of potato haulm killing, increasing the resolution from $30 \times 30 \mathrm{~m}^{2}$ to just $15 \times 15 \mathrm{~m}^{2}$ will reduce herbicide use from 2.2 to $1.7 \mathrm{~L} \mathrm{ha}^{-1}$ [15] which is a reduction of $60 \%$ relative to conventional application. Similarly, for soil herbicides a reduction of $50 \%$ and for late blight a reduction of $40 \%$ seems possible (relative to conventional application). For sidedress $\mathrm{N}$ a further reduction in $\mathrm{N}$ use is not possible, but it is expected that real-time soil $\mathrm{NO}_{3}$ measurements using new sensors will allow further reductions in $\mathrm{N}$ use.

Supplementary Materials: The following are available online at www.mdpi.com/2071-1050/9/10/1863/s1. Table S1: Profit and social profit of potato production under standard production and under four precision agriculture scenarios, Table S2: Profit and social profit of olive production under standard production and under precision agriculture.

Acknowledgments: Precision agriculture research in The Netherlands is often performed in public-private partnership projects. C.K. and F.K.v.E. gratefully acknowledge the many parties we worked with in the last 10 years to collect all the data presented. The work could not have been done without technical support by Agrometius, Bayer, Homburg Holland, Kverneland, LTO Noord, Syngenta, Yara, and ZLTO, and the IJKakker and PL2.0 partners (see www.precisielandbouw.eu). We thank the farmers on the many test locations for allowing us to do the field experiments.

Author Contributions: F.K.v.E. conceived the overall setup of the paper and contributed data on N sidedress trials in potatoes. S.F. contributed data on olives and contributed to the economic analysis. D.G.-C. contributed to the economic and social profit analyses of potatoes and olives. C.K. contributed data on soil herbicide, late blight 
and potato haulm killing trials, as well as to the economic analysis of potato production. All authors contributed to the Discussion section.

Conflicts of Interest: The authors declare no conflict of interest.

Disclaimer: The fact that some products are mentioned by name does not imply endorsement of these products by the authors.

$\begin{array}{ll}\text { Abbreviations } \\ \text { CTF } & \text { Controlled Traffic Farming } \\ \text { a.i. } & \text { Active ingredient(s) } \\ \text { EIQ } & \text { Environmental Impact Quotient } \\ \text { GHG } & \text { Greenhouse gases } \\ \text { GNSS } & \text { Global Navigation Satellite System } \\ \text { FMIS } & \text { Farm Management Information System } \\ \text { KWIN } & \text { Kwantitatieve Informatie (“Quantitative Information”) } \\ \text { LCA } & \text { Life Cycle Analysis } \\ \text { PA } & \text { Precision Agriculture } \\ \text { UAV } & \text { Unmanned Aerial Vehicle } \\ \text { VRA } & \text { Variable Rate Application }\end{array}$

\section{References}

1. Foley, J.A.; Ramankutty, N.; Brauman, K.A.; Cassidy, E.S.; Gerber, J.S.; Johnston, M.; Mueller, N.D.; O'Connell, C.; Ray, D.K.; West, P.C.; et al. Solutions for a cultivated planet. Nature 2011, 478, 337-342. [CrossRef] [PubMed]

2. Basso, B.; Dumont, B.; Cammarano, D.; Pezzuolo, A.; Marinello, F.; Sartori, L. Environmental and economic benefits of variable rate nitrogen fertilization in a nitrate vulnerable zone. Sci. Total Environ. 2016, 545-546, 227-235. [CrossRef] [PubMed]

3. Fleming, K.L.; Westfall, D.G.; Wiens, D.W.; Brodahl, M.C. Evaluating farmer defined management zone maps for variable rate fertilizer application. Precis. Agric. 2000, 2, 201-215. [CrossRef]

4. Schwarz, J.; Herold, L.; Pollin, B. Typology of PF Technologies; FP7 Project Future Farm. Available online: www.futurefarm.eu (accessed on 20 August 2017).

5. Cho, Y.; Sudduth, K.A.; Chung, S.O. Soil physical property estimation from soil strength and apparent electrical conductivity sensor data. Biosyst. Eng. 2016, 152, 68-78. [CrossRef]

6. Duan, T.; Chapman, S.C.; Guo, Y.; Zheng, B. Dynamic monitoring of ndvi in wheat agronomy and breeding trials using an unmanned aerial vehicle. Field Crops Res. 2017, 210, 71-80. [CrossRef]

7. Holpp, M.; Kroulik, M.; Kviz, Z.; Anken, T.; Sauter, M.; Hensel, O. Large-scale field evaluation of driving performance and ergonomic effects of satellite-based guidance systems. Biosyst. Eng. 2013, 116, 190-197. [CrossRef]

8. Fountas, S.; Carli, G.; Sorensen, C.G.; Tsiropoulos, Z.; Cavalaris, C.; Vatsanidou, A.; Liakos, B.; Canavari, M.; Wiebensohn, J.; Tisserye, B. Farm management information systems: Current situation and future perspectives. Comput. Electron. Agric. 2015, 115, 40-50. [CrossRef]

9. Been, T.H.; Schomaker, C.H.; Molendijk, L.P.G. Nemadecide, a decision support system for the management of potato cyst nematodes. Phytopathology 2007, 97, S152.

10. Been, T.H.; Schomaker, C.H. A geo-referenced decision support system for nematodes in potatoes. In Decision Support Systems in Potato Production: Bringing Models to Practice; MacKerron, D.K.L., Haverkort, A.J., Eds.; Wageningen Academic Publishers: Wageningen, The Netherlands, 2004; pp. 154-167.

11. Kempenaar, C.; Van Evert, F.K.; Been, T. Use of Vegetation Indices in Variable Rate Application of Potato Haulm Killing Herbicides. In Proceedings of the 12th International Conference on Precision Agriculture (ICPA), Sacramento, CA, USA, 20-23 July 2014.

12. Kempenaar, C.; Van Evert, F.K.; Been, T.; Kocks, C.G.; Westerdijk, C.E. Towards Data-Intensive, More Sustainable Farming: Advances in Predicting Crop Growth and Use of Variable Rate Technology in Arable Crops in the Netherlands. In Proceedings of the 13th International Conference on Precision Agriculture (ICPA), St. Louis, MO, USA, 31 July-3 August 2016. 
13. Van Evert, F.K.; Fountas, S.; Jakovetic, D.; Crnojevic, V.; Travlos, I.; Kempenaar, C. Big data for weed control and crop protection. Weed Res. 2017, 57, 218-233. [CrossRef]

14. Christensen, S.; Sogaard, H.T.; Kudsk, P.; Norremark, M.; Lund, I.; Nadimi, E.S.; Jorgensen, R. Site-specific weed control technologies. Weed Res. 2009, 49, 233-241. [CrossRef]

15. Van Evert, F.K.; Van der Voet, P.; Van Valkengoed, E.; Kooistra, L.; Kempenaar, C. Satellite-based herbicide rate recommendation for potato haulm killing. Eur. J. Agron. 2012, 43, 47-57. [CrossRef]

16. Parsons, D.J.; Benjamin, L.; Clarke, J.; Ginsburg, D.; Mayes, A.; Milne, A.; Wilkinson, D. Weed manager-A model-based decision support system for weed management in arable crops. Comput. Electron. Agric. 2009, 65, 155-167. [CrossRef]

17. Rydahl, P. A Danish Decision Support System for Integrated Management of Weeds. Asp. Appl. Biol. Adv. Appl. Biol. Provid. New Oppor. Consum. Prod. 21st Century 2004, 72, 43-53.

18. Cooke, L.R.; Schepers, H.T.A.M.; Hermansen, A.; Bain, R.A.; Bradshaw, N.J.; Ritchie, F.; Shaw, D.S.; Evenhuis, A.; Kessel, G.J.T.; Wander, J.G.N.; et al. Epidemiology and integrated control of potato late blight in europe. Potato Res. 2011, 54, 183-222. [CrossRef]

19. World Commission on Environment and Development. Our Common Future; Oxford University Press: Oxford, UK, 1987.

20. World Bank. Environment, Growth, and Development. Development Committee Pamphlet 14; World Bank: Washington, DC, USA, 1987.

21. Clark, W.C.; Munn, R.E. Sustainable Development of the Biosphere; Cambridge University Press: Cambridge, UK, 1986.

22. Elkington, J. Triple bottom line revolution: Reporting for the third millennium. Aust. CPA 1999, 69, 75-76.

23. Pearce, D.W.; Atkinson, G.D. Capital theory and the measurement of sustainable development: An indicator of "weak" sustainability. Ecol. Econ. 1993, 8, 103-108. [CrossRef]

24. Repetto, R. The Global Possible: Resources, Development, and the New Century; Yale University Press: New Haven, CT, USA, 1986.

25. Elkington, J. Towards the sustainable corporation-win-win-win business strategies for sustainable development. Calif. Manag. Rev. 1994, 36, 90-100. [CrossRef]

26. Elkington, J. Cannibals with Forks; Capstone: Oxford, UK, 1997.

27. Gomez-Limon, J.A.; Sanchez-Fernandez, G. Empirical evaluation of agricultural sustainability using composite indicators. Ecol. Econ. 2010, 69, 1062-1075. [CrossRef]

28. Haas, G.; Wetterich, F.; Köpke, U. Comparing intensive, extensified and organic grassland farming in southern germany by process life cycle assessment. Agric. Ecosyst. Environ. 2001, 83, 43-53. [CrossRef]

29. Foteinis, S.; Chatzisymeon, E. Life cycle assessment of organic versus conventional agriculture. A case study of lettuce cultivation in greece. J. Clean. Prod. 2016, 112, 2462-2471. [CrossRef]

30. Cambria, D.; Vázquez-Rowe, I.; González-García, S.; Moreira, M.T.; Feijoo, G.; Pierangeli, D. Comparative life cycle assessment study of three winter wheat production systems in the european union. Environ. Eng. Manag. J. (EEMJ) 2016, 51, 1755-1766.

31. Bockstaller, C.; Girardin, P.; van der Werf, H.M.G. Use of agro-ecological indicators for the evaluation of farming systems. Eur. J. Agron. 1997, 7, 261-270. [CrossRef]

32. Bockstaller, C.; Guichard, L.; Keichinger, O.; Girardin, P.; Galan, M.B.; Gaillard, G. Comparison of methods to assess the sustainability of agricultural systems: A review. Agron. Sustain. Dev. 2009, 29, 223-235. [CrossRef]

33. Van Asselt, E.D.; Van Bussel, L.G.J.; Van der Voet, H.; Van der Heijden, G.W.A.M.; Tromp, S.O.; Rijgersberg, H.; Van Evert, F.; Van Wagenberg, C.P.A.; Van der Fels-Klerx, H.J. A protocol for evaluating the sustainability of agri-food production systems-A case study on potato production in peri-urban agriculture in the netherlands ecological indicators. Accept. Ecol. Indic. 2014, 43, 315-321. [CrossRef]

34. Färe, R.; Grosskopf, S.; Lundgren, T.; Marklund, P.-O.; Zhou, W. Productivity: Should We Include Bads? CERE (Center for Environmental and Resource Economics) Working Paper 2012:13; Umeå University: Umeå, Sweden, 2012; Available online: http:/ / dx.doi.org/10.2139/ssrn.2071078 (accessed on 14 October 2017).

35. Glendining, M.J.; Dailey, A.G.; Williams, A.G.; Van Evert, F.K.; Goulding, K.W.T.; Whitmore, A.P. Is it possible to increase the sustainability of arable and ruminant agriculture by reducing inputs? Agric. Syst. 2009, 99, 117-125. [CrossRef]

36. Barnett, V.; Payne, R.; Steiner, R. Agricultural Sustainability: Economic, Environmental and Statistical Considerations; John Wiley and Sons: Chicester, UK, 1995. 
37. Gaitán-Cremaschi, D.; Kamali, F.P.; van Evert, F.K.; Meuwissen, M.P.M.; Oude Lansink, A.G.J.M. Benchmarking the sustainability performance of the brazilian non-gm and gm soybean meal chains: An indicator-based approach. Food Policy 2015, 55, 22-32. [CrossRef]

38. Taylor, D.C.; Mohamed, Z.A.; Shamsudin, M.N.; Mohayidin, M.G.; Chiew, E.F. Creating a farmer sustainability index: A malaysian case study. Am. J. Altern. Agric. 1993, 8, 175-184. [CrossRef]

39. Wackernagel, M.; Rees, W. Our Ecological Footprint: Reducing Human Impact on the Earth; New Society Publishers: Gabriola Island, BC, Canada, 1998.

40. Gaitán Cremaschi, D.; Pashaei Kamali, F.; van Evert, F.K.; Meuwissen, M.P.M.; Oude Lansink, A.G.J.M. Benchmarking the Sustainability of Soybean Meal Production in Brazil Using a Total Factor Productivity Approach. In Proceedings of the 29th International Conference of Agricultural Economists, Milan, Italy, 8-14 August 2015.

41. Nordhaus, W.D. Revisiting the social cost of carbon. Proc. Natl. Acad. Sci. USA 2017, 114, 1518-1523. [CrossRef] [PubMed]

42. Silalertruksa, T.; Bonnet, S.; Gheewala, S.H. Life cycle costing and externalities of palm oil biodiesel in thailand. J. Clean. Prod. 2012, 28, 225-232. [CrossRef]

43. Kovach, J.; Petzoldt, C.; Degni, J.; Tette, J. A method to measure the environmental impact of pesticides. New York's Food and Life Sciences Bulletin 139. Cornel. Digit. Repos. 1992. Available online: http: / / hdl.handle.net/1813/5203 (accessed on 14 October 2017).

44. Praneetvatakul, S.; Schreinemachers, P.; Pananurak, P.; Tipraqsa, P. Pesticides, external costs and policy options for thai agriculture. Environ. Sci. Policy 2013, 27, 103-113. [CrossRef]

45. Pretty, J.; Heffron, L.; Nedwell, D. The Costs and Benefits of Nitrogen Enrichment. The NERC Global Nitrogen Enrichment (GANE) Programme Finale; Royal Geographical Society: London, UK, 2005. Available online: http:/ / webarchive.nationalarchives.gov.uk/20140102180316/http:/ / www.nerc.ac.uk/research/ programmes/gane/results/documents/pretty.pdf (accessed on 14 October 2017).

46. Pretty, J.N.; Ball, A.S.; Lang, T.; Morison, J.I. Farm costs and food miles: An assessment of the full cost of the uk weekly food basket. Food Policy 2005, 30, 1-19. [CrossRef]

47. Pretty, J.N.; Brett, C.; Gee, D.; Hine, R.E.; Mason, C.F.; Morison, J.I.L.; Raven, H.; Rayment, M.D.; van der Bijl, G. An assessment of the total external costs of uk agriculture. Agric. Syst. 2000, 65, 113-136. [CrossRef]

48. Pretty, J.N.; Mason, C.F.; Nedwell, D.B.; Hine, R.E.; Leaf, S.; Dils, R. Environmental costs of freshwater eutrophication in england and wales. Environ. Sci. Technol. 2003, 37, 201-208. [CrossRef] [PubMed]

49. Haverkort, A.J.; Boonekamp, P.J.; Hutten, R.; Jacobsen, E.; Lotz, L.A.P.; Kessel, G.T.J.; Visser, R.G.F.; Van der Vossen, E.A.G. Societal costs of late blight in potato and prospects of durable resistance through cisgenic modification. Potato Res. 2008, 51, 47-57. [CrossRef]

50. Praktijkonderzoek Plant en Omgeving. Kwantitatieve Informatie Akkerbouw en Vollegrondsgroenteteelt 2015; Praktijkonderzoek Plant \& Omgeving B.V.: Lelystad, The Netherlands, 2015.

51. Proefstation voor de Akkerbouw en de Groenteteelt in de Vollegrond. Kwantitatieve Informatie Voor de Akkerbouw en de Groenteteelt in de Vollegrond 1995; Proefstation voor de Akkerbouw en de Groenteteelt in de Vollegrond (PAGV): Lelystad, The Netherlands, 1995.

52. Skelsey, P.; Kessel, G.; Holtslag, A.; Moene, A.; Van Der Werf, W. Regional spore dispersal as a factor in disease risk warnings for potato late blight: A proof of concept. Agric. For. Meteorol. 2009, 149, 419-430. [CrossRef]

53. Kempenaar, C.; Oosterhuis, H.S.; Van der Lans, A.M.; Van der Schans, D.A.; Stilma, E.S.C.; Hendriks-Goossens, V.J.C.; Verwijs, B.R.; Van Wijk, C.A.P.; Van de Zande, J.C.; Lotz, L.A.P. Ontwikkeling van Het Prototype Sensispray in de Gewassen Aardappel en Tulp. Nota 667; Plant Research International: Wageningen, The Netherlands, 2010.

54. Van Dijk, W.; Van Geel, W. Adviesbasis voor de Bemesting Van Akkerbouwgewassen = Fertilizer Recommendations for Arable Crops; Praktijkonderzoek Plant \& Omgeving (PPO): Lelystad, The Netherlands, 2012; Available online: http:/ / www.kennisakker.nl/files/Adviesbasis_januari_2012.pdf (accessed on 14 October 2017).

55. Samborski, S.M.; Tremblay, N.; Fallon, E. Strategies to make use of plant sensors-based diagnostic information for nitrogen recommendations. Agron. J. 2009, 101, 800-816. [CrossRef]

56. Booij, R.; Uenk, D.; Lokhorst, C.; Sonneveld, C. Monitoring crop nitrogen status in potatoes, using crop light reflection. In Proceedings of the Third European Conference on Precision Agriculture, Montpellier, France, 18-20 June 2001; pp. 893-899. 
57. Van Evert, F.K.; Booij, R.; Jukema, J.N.; Ten Berge, H.F.M.; Uenk, D.; Meurs, E.J.J.; Van Geel, W.C.A.; Wijnholds, K.H.; Slabbekoorn, H. Using crop reflectance to determine sidedress $\mathrm{n}$ rate in potato saves $\mathrm{n}$ and maintains yield. Eur. J. Agron. 2012, 43, 58-67. [CrossRef]

58. Kempenaar, C.; Struijk, P.C. The canon of potato science: Haulm killing. Potato Res. 2008, 50, 341-345. [CrossRef]

59. Heijting, S.; Kempenaar, C. Perspectives for site-specific application of soil herbicides in arable farming. Gewasbescherming 2013, 44, 139.

60. Jongema, M. Inhoudelijk Eindverslag IJkakker. LTO Noord, Zwolle. 2016. Available online: http:/ / www. Precisielandbouw.Eu/dossiers/119-ijkakker (accessed on 14 October 2017).

61. Blok, P.; Kempenaar, C. Validatie Toepassingen Sensispray in Aardappelen en Wintertarwe in 2011: Variabel Doseren van Enkele Fungiciden, een Vloeibare N-Meststof, een Groeiregulator en een Loofdoodmiddel; Wageningen University \& Research: Wageningen, The Netherlands, 2011.

62. Kempenaar, C.; Heijting, S.; Kessel, G.J.T.; Michielsen, J.G.P.; Wijnholds, K.H. Modellen en Beslisregels voor Variabel Doseren van Gewasbeschermingsmiddelen op Basis van Variatie in Bodem en Gewas; Plant Research International: Wageningen, The Netherlands, 2013.

63. Van Evert, F.K.; Van der Schans, D.A.; Van Geel, W.C.A.; Malda, J.T.; Vona, V. From Theory to Practice: Using Canopy Reflectance to Determine Sidedress $n$ Rate in Potatoes. In Proceedings of the 9th European Conference on Precision Agriculture (ECPA 2013), Lleida, Spain, 7-11 July 2013; Stafford, J.V., Ed.; Wageningen Academic Publishers: Lleida, Spain, 2013; pp. 119-127.

64. Jongschaap, R.E.E.; Booij, R. Spectral measurements at different spatial scales in potato: Relating leaf, plant and canopy nitrogen status. Int. J. Appl. Earth Obs. Geoinf. 2004, 5, 205-218. [CrossRef]

65. van Geel, W.C.A.; Meurs, E.J.J. Ontwikkeling Geleide Bemestingssystemen in de Teelt van Prei 2002-2003; Praktijkonderzoek Plant \& Omgeving, Akkerbouw, Groene Ruimte en Vollegrondsgroenten: Lelystad, The Netherlands, 2004.

66. Slabbekoorn, H. Stikstofbijmestsystemen in Consumptieaardappelen, 2002 (N Sidedress Systems in Ware Potatoes, 2002); WUR-PPO: Westmaas, The Netherlands, 2002.

67. Slabbekoorn, H. Stikstofbijmestsystemen in Consumptieaardappelen, 2003 (N Sidedress Systems in Ware Potatoes, 2003); WUR-PPO: Westmaas, The Netherlands, 2003.

68. Van Evert, F.K.; Van der Schans, D.A.; Malda, J.T.; Van den Berg, W.; Van Geel, W.C.A.; Jukema, J.N. Geleide N-Bemesting voor Aardappelen op Basis van Gewasreflectie-Metingen: Integratie van Sensormetingen in Een N-Bijmestsysteem; PPO Rapport 423; Praktijkonderzoek Plant \& Omgeving (PPO): Lelystad, The Netherlands, 2011; Volume 423.

69. Van der Schans, D.A.; Van Evert, F.K.; Malda, J.T.; Dorka-Vona, V. Sensorgestuurde Advisering van Stikstof Bijbemesting in Aardappel: Implementatie en Integratie; PPO Rapport 520; Praktijkonderzoek Plant \& Omgeving (PPO): Wageningen, The Netherlands, 2012.

70. FAOSTAT. Olive Production in Greece; Food and Agriculture Organization of the United Nations: Rome, Italy, 2017; Retrieved 28 July 2017; Available online: http: / / www.Fao.Org/faostat (accessed on 16 October 2017).

71. Fountas, S.; Aggelopoulou, K.; Bouloulis, C.; Nanos, G.D.; Wulfsohn, D.; Gemtos, T.A.; Paraskevopoulos, A.; Galanis, M. Site-specific management in an olive tree plantation. Precis. Agric. 2011, 12, 179-195. [CrossRef]

72. Russo, C.; Cappelletti, G.M.; Nicoletti, G.M.; Di Noia, A.E.; Michalopoulos, G. Comparison of European olive production systems. Sustainability 2016, 8, 825. [CrossRef]

73. Haverkort, A.J.; Hillier, J.G. Cool farm tool - potato: Model description and performance of four production systems. Potato Res. 2011, 54, 355-369. [CrossRef]

74. Monteith, J. Can sustainability be quantified? Indian J. Dryland Agric. Res. Dev. 1990, 5, 1-15.

75. Hansen, J.W.; Jones, J.W. A systems framework for characterizing farm sustainability. Agric. Syst. 1996, 51, 185-201. [CrossRef]

76. Harwood, R.R. A history of sustainable agriculture. In Sustainable agricultural systems; Edwards, C.A., Lal, R., Madden, P., Eds.; Soil and Water Conservation Society: Ankeny, IA, USA, 1990; pp. 3-19.

77. Sinha, E.; Michalak, A.M.; Balaji, V. Eutrophication will increase during the 21st century as a result of precipitation changes. Science 2017, 357, 405-408. [CrossRef] [PubMed]

78. Kniss, A.R.; Coburn, C.W. Quantitative evaluation of the environmental impact quotient (eiq) for comparing herbicides. PLoS ONE 2015, 10, e0131200. [CrossRef] [PubMed] 
79. Dalal-Clayton, B.; Sadler, B. Sustainability Appraisal: A Sourcebook and Reference Guide to International Experience; Routledge: Abingdon, UK, 2014.

80. Timmer, R.D.; Wustman, R. Onderbouwing Van de Effecten van een Variabele Plantafstand Bij Pootaardappelen; Praktijkonderzoek Plant \& Omgeving, Business Unit AGV: Lelystad, The Netherlands, 2007; Available online: http: / / edepot.wur.nl/180866 (accessed on 14 October 2017).

81. Malda, J.T.; Specken, J. Het Effect van de Pootafstand op Percelen Met Variatie in Lutumgehalte in de Teelt van Tafelaardappelen in 2010; Altic: Dronten, The Netherlands, 2011; Available online: http:/ / edepot.wur.nl/ 299776 (accessed on 14 October 2017).

C 2017 by the authors. Licensee MDPI, Basel, Switzerland. This article is an open access article distributed under the terms and conditions of the Creative Commons Attribution (CC BY) license (http:/ / creativecommons.org/licenses/by/4.0/). 